\title{
Supporting Information (I)
}

\section{Intramolecular Nitrile Oxide-Alkene Cycloaddition of sugar derivatives with unmasked hydroxyl group(s)}

Tony K. M. Shing, ${ }^{*}$ Wai F. Wong, Hau M. Cheng, Wun S. Kwok, King H. So

Department of Chemistry and Center of Novel Functional Molecules, The Chinese University of

Hong Kong, Shatin, NT, Hong Kong

E-mail: tonyshing@cuhk.edu.hk

RECEIVED DATE (automatically inserted by publisher);

Appendices

S2-S3 Product characterization

S4-S20 Experimental procedures.

S21-S24 X-ray crystallographic structure of 14a, 18a, 21 and 24. 


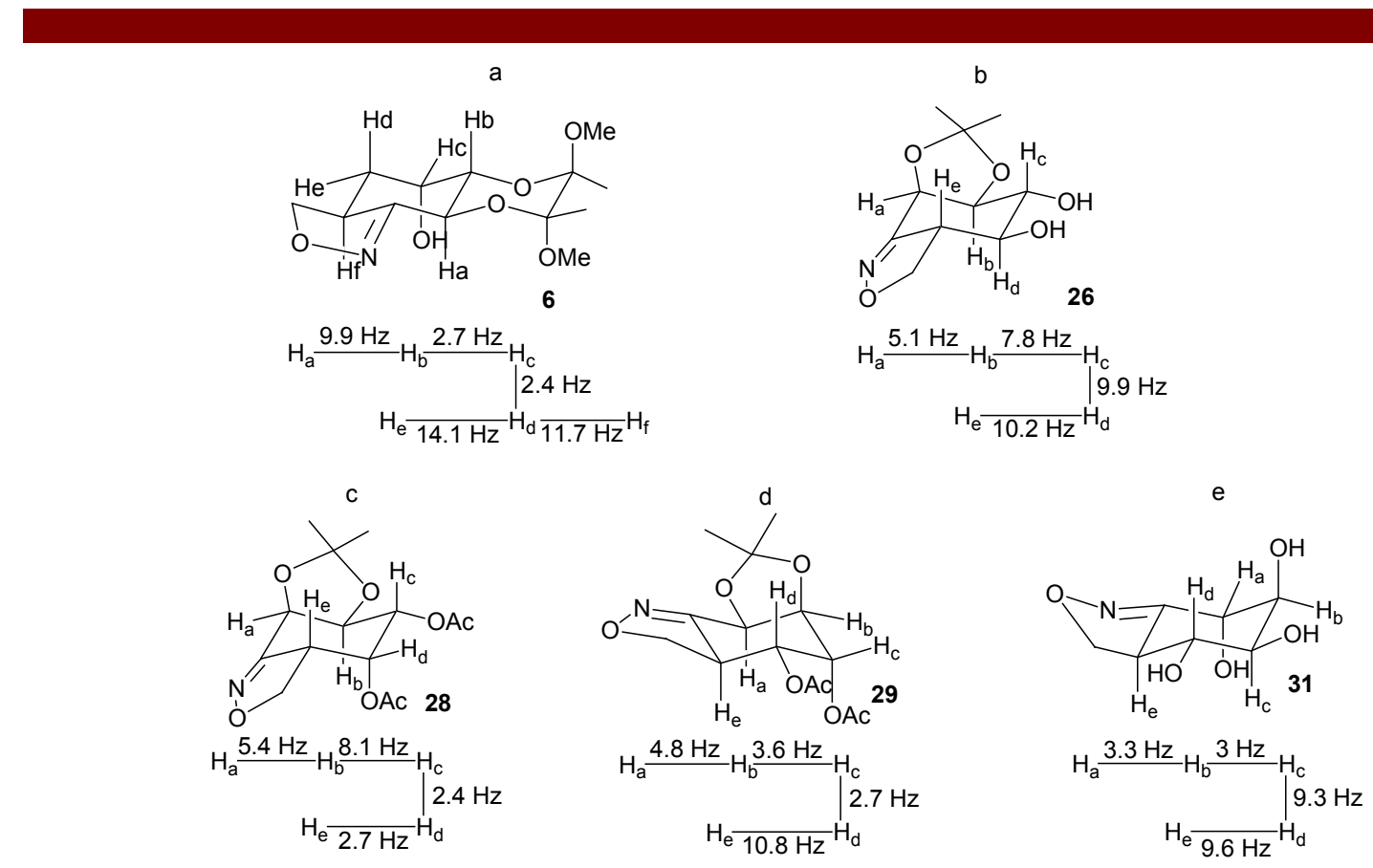

Figure 1. Conformation of isoxazolines 6, 26, 28, 29 and 31

Single crystals of isoxazolines $\mathbf{2 1}$ and $\mathbf{2 4}$ and acetate derivative of isoxazolines $\mathbf{1 4}$ and $\mathbf{1 8}$ confirmed their constitution by X-ray crystallography. By exclusion, structures of cyclohexanes 19 and 22 were assigned. The ${ }^{1} \mathrm{H}$ and ${ }^{13} \mathrm{C}$ NMR spectra of enantiomeric cycloadducts $\mathbf{1 1}, \mathbf{1 2}$ and $\mathbf{1 6}$ were compared with that of 18, 19 and 14, respectively for structural confirmation. The stereochemistry of isoxazolines $\mathbf{8}$ and $\mathbf{1 0}$ were confirmed by comparing the optical rotation, melting point and the ${ }^{1} \mathrm{H}$ and ${ }^{13} \mathrm{C}$ NMR spectroscopic data of their acetate derivatives with the published data $^{8,9 a}$ and personal communication ${ }^{9 b-d}$ provided by Professor V. Jäger.

The structure of cyclohexane 6 could be readily assigned by ${ }^{1} \mathrm{H}$ NMR analysis (Figure 1a). Due to the rigidity of the diacetal ring, $\mathrm{H}_{\mathrm{a}}$ and $\mathrm{H}_{\mathrm{b}}$ occupied the axial position with coupling constant $J=$ 10.2 Hz. $\mathrm{H}_{\mathrm{c}}$ was a quartet with coupling constant $J=2.7 \mathrm{~Hz}$ which fell into the range of equatorialequatorial or equatorial-axial coupling. $H_{c}$ should therefore in the equatorial position and $H_{d}$ in the axial position. The large coupling constant $\left(J_{\mathrm{d}, \mathrm{f}}=11.7 \mathrm{~Hz}\right)$ between $\mathrm{H}_{\mathrm{d}}$ and $\mathrm{H}_{\mathrm{f}}$ suggested a diaxial arrangement which confirmed that the isoxazoline ring was bending to the $\beta$ face. For isoxazoline 26 (Figure 1b), the trans-diaxial arrangements of $\mathrm{H}_{\mathrm{b}}, \mathrm{H}_{\mathrm{c}}$ and $\mathrm{H}_{\mathrm{d}}$ were supported by the large coupling 
constants $(J=7.8-9.9 \mathrm{~Hz})$. The large axial-axial coupling of $\mathrm{H}_{\mathrm{d}}$ with $\mathrm{H}_{\mathrm{e}}\left(J_{\mathrm{d}, \mathrm{e}}=10.2 \mathrm{~Hz}\right)$ indicated the heterocyclic ring was bending to the $\alpha$ face.

In Figure 1c, the diaxial arrangement of $\mathrm{H}_{\mathrm{b}}$ and $\mathrm{H}_{\mathrm{c}}$ was supported by the large coupling constant $\left(J_{\mathrm{b}, \mathrm{c}}=8.1 \mathrm{~Hz}\right)$. The axial-equatorial couplings of $\mathrm{H}_{\mathrm{c}}$ with $\mathrm{H}_{\mathrm{d}}\left(J_{\mathrm{c}, \mathrm{d}}=2.4 \mathrm{~Hz}\right)$ and $\mathrm{H}_{\mathrm{d}}$ with $\mathrm{H}_{\mathrm{e}}\left(J_{\mathrm{d}, \mathrm{e}}=2.7\right.$ $\mathrm{Hz}$ ) verified the configuration of isozaxoline 28. Concerning the structure of diastereomeric cycloadduct 29 (Figure 1d), the small coupling constant of $\mathrm{H}_{\mathrm{b}}$ with $\mathrm{H}_{\mathrm{c}}\left(J_{\mathrm{b}, \mathrm{c}}=3.6 \mathrm{~Hz}\right)$ fell into the range of equatorial-equatorial coupling and the smaller coupling constant between $\mathrm{H}_{\mathrm{c}}$ and $\mathrm{H}_{\mathrm{d}}\left(J_{\mathrm{c}, \mathrm{d}}=\right.$ $2.7 \mathrm{~Hz}$ ) demonstrated an axial-equatorial arrangement. The large coupling constant of $\mathrm{H}_{\mathrm{d}}$ with $\mathrm{H}_{\mathrm{e}}$ $\left(J_{\mathrm{d}, \mathrm{e}}=10.8 \mathrm{~Hz}\right)$ suggested a $\beta$ assignment of the heterocyclic ring.

For cycloadduct 31 (Figure 1e), the small coupling constants of $\mathrm{H}_{\mathrm{a}}$ with $\mathrm{H}_{\mathrm{b}}\left(J_{\mathrm{a}, \mathrm{b}}=3.3 \mathrm{~Hz}\right)$ and $\mathrm{H}_{\mathrm{b}}$ with $\mathrm{H}_{\mathrm{c}}\left(J_{\mathrm{b}, \mathrm{c}}=3 \mathrm{~Hz}\right)$ suggested an equatorial deployment of $\mathrm{H}_{\mathrm{b}}$. The large coupling constants of $\mathrm{H}_{\mathrm{c}}$ with $\mathrm{H}_{\mathrm{d}}\left(J_{\mathrm{c}, \mathrm{d}}=9.3 \mathrm{~Hz}\right)$ and $\mathrm{H}_{\mathrm{d}}$ with $\mathrm{H}_{\mathrm{e}}\left(J_{\mathrm{d}, \mathrm{e}}=9.6 \mathrm{~Hz}\right)$ demonstrates the heterocyclic ring was bending to the $\beta$ face. 


\section{Experimental Section}

Melting points are uncorrected. Optical rotations were obtained with a polarimeter operating at 589nm. Infrared spectra (IR) were recorded with a FT-IR spectrophotometer as thin film on potassium bromide discs. Nuclear magnetic resonance (NMR) spectra were measured at 300.13 $\mathrm{MHz}\left({ }^{1} \mathrm{H}\right)$ or at $75.47 \mathrm{MHz}\left({ }^{13} \mathrm{C}\right)$ in $\mathrm{CDCl}_{3}$ solutions, unless stated otherwise. All chemical shifts were recorded in ppm relative to tetramethylsilane $(\delta=0.0)$. Spin-spin coupling constants ( $J$ value $)$ recorded in $\mathrm{Hz}$ were measured directly from the spectra. All reactions were monitored by analytical thin-layer chromatography (TLC) on aluminium-precoated plates of silica gel with detection by spraying with $5 \%(\mathrm{w} / \mathrm{v})$ dodecamolybdophosphoric acid in ethanol or $5 \%(\mathrm{w} / \mathrm{v})$ ninhydrin in ethanol and subsequent heating. Silica gel (230-400 mesh) was used for flash chromatography. All reagents and solvents were general reagent grade unless otherwise stated. 2-Propanol was dried by sodium and distilled from its sodium salt under nitrogen. DMF was dried by magnesium sulfate, filtered and was then freshly distilled under reduced pressure. Acetonitrile was freshly distilled from $\mathrm{P}_{2} \mathrm{O}_{5}$ under nitrogen. THF was freshly distilled from $\mathrm{Na} /$ benzophenone ketyl under nitrogen. Dichloromethane and chloroform were freshly distilled from $\mathrm{P}_{2} \mathrm{O}_{5}$ under nitrogen. Diethyl ether was freshly distilled from $\mathrm{K} /$ benzophenone ketyl under nitrogen. Other reagents were purchased from commercial suppliers and were used without purification.

Activation of $\mathbf{Z n}$ powder. ${ }^{15 \mathrm{a}} \mathrm{Zn}$ powder was stirred in $2 \% \mathrm{HCl}$ at room temperature for $30 \mathrm{~min}$. The activated $\mathrm{Zn}$ powder was filtered and washed with deionized $\mathrm{H}_{2} \mathrm{O}, \mathrm{EtOH}$, and then $\mathrm{Et}_{2} \mathrm{O}$. Any $\mathrm{Et}_{2} \mathrm{O}$ residue was removed under vacuum and the $\mathrm{Zn}$ powder was then flame dried in nitrogen and cooled down before use.

Generation of allylmagnesium bromide solution. To a suspension of magnesium powder (3.65 $\mathrm{g}, 150 \mathrm{mmol})$ in $\mathrm{Et}_{2} \mathrm{O}(10 \mathrm{~mL})$ was added a catalytic amount of iodine and the mixture was stirred at room temperature for $15 \mathrm{~min}$. A solution of allyl bromide $(4.30 \mathrm{~mL}, 50.0 \mathrm{mmol})$ in $\mathrm{Et}_{2} \mathrm{O}(50 \mathrm{~mL})$ was added dropwise to the mixture at a rate to maintain a gentle reflux. After the addition of the allyl bromide solution, the mixture was heated under reflux for $30 \mathrm{~min}$ and then cooled down. The concentration of the allylmagnesium bromide solution generated was about $1.5 \mathrm{M}$. 
Generation of vinylmagnesium bromide solution. Vinyl bromide $(1.6 \mathrm{~mL}, 95.0 \mathrm{mmol})$ was condensed with a cold finger and diluted with THF $(30 \mathrm{~mL})$ at $-78{ }^{\circ} \mathrm{C}$. To a suspension of magnesium powder $(7.4 \mathrm{~g}, 304.5 \mathrm{mmol})$ in THF $(30 \mathrm{~mL})$ was added 1,2-dibromoethane $(1 \mathrm{~mL})$ and the vinyl bromide solution was added dropwise to the reaction mixture at a rate to maintain a moderate reflux. After the addition was completed, the solution was heated under reflux for $30 \mathrm{~min}$ and then cooled down. The concentration of the vinylmagnesium bromide solution generated was about $1.5 \mathrm{M}$. The concentration of the vinylmagnesium bromide solution can be determined by forming indicator with 1,10-phenanthroline, which gives a bright violet solution. Then adding $1 \mathrm{M}$ sec-butyl alcohol in xylene as titrant until the color fades out sharply.

General procedure for glycol oxidative cleavage. $\mathrm{NaIO}_{4}(3 \mathrm{mmol})$ was dissolved in a minimum amount of hot water $\left(\sim 80{ }^{\circ} \mathrm{C}, 3 \mathrm{~mL}\right)$ and to this solution was added silica gel (230-400 mesh, $10 \times$ weight of diol) with vigorous swirling and shaking. The mixture was suspended in $\mathrm{CH}_{2} \mathrm{Cl}_{2}(10 \mathrm{~mL})$ and then a solution of diol $(1 \mathrm{mmol})$ in $\mathrm{CH}_{2} \mathrm{Cl}_{2}(10 \mathrm{~mL})$ was added. After vigorous stirring at room temperature for $1 \mathrm{~h}$, the mixture was filtered. The filtrate was concentrated under reduced pressure to give the aldehyde product.

General procedure for the formation of oxime (method A). Hydroxylamine hydrochloride (1.5 mmol) and $\mathrm{NaHCO}_{3}(2 \mathrm{mmol})$ were added to a solution of the aldehyde in $\mathrm{MeOH}(10 \mathrm{~mL})$. The reaction mixture was stirred at room temperature until the disappearance of the aldehyde as shown on TLC and was then partitioned between EtOAc $(15 \mathrm{~mL})$ and water $(15 \mathrm{~mL})$. The aqueous layer was extracted with EtOAc (15 mL x 3). The combined organic extracts were washed with brine (10 $\mathrm{mL}$ ), dried over anhydrous $\mathrm{MgSO}_{4}$, and filtered. Concentration of the filtrate gave the oxime.

General procedure for the formation of oxime (method B). Hydroxylamine hydrochloride (1.5 $\mathrm{mmol})$ was added to a solution of the aldehyde in pyridine $(10 \mathrm{~mL})$. The reaction mixture was stirred at room temperature until the disappearance of the aldehyde as shown on TLC. Concentration of the solvent under reduced pressure and then purification with flash chromatography to remove pyridinium chloride gave the oxime. 
General procedure for the INOC reaction with chloramine-T and silica gel (method I). To a stirred suspension of the oxime ( $1 \mathrm{mmol})$ and silica gel (230-400 mesh, $5 \times$ weight of diol) in EtOH $(10 \mathrm{~mL})$ was added dropwise a milky solution of chloramine-T trihydrate $(1.5 \mathrm{mmol})$ in $\mathrm{EtOH}(5$ $\mathrm{mL}$ ). The reaction mixture was stirred at room temperature until the disappearance of the oxime as shown on TLC and the silica gel was filtered off. Concentration of the filtrate followed by flash chromatography of the residue afforded the isoxazoline.

General procedure for the INOC reaction with chloramine-T without silica gel (method II). To a stirred suspension of the oxime $(1 \mathrm{mmol})$ in $\mathrm{EtOH}(10 \mathrm{~mL})$ was added dropwise a milky solution of chloramine-T trihydrate $(1.5 \mathrm{mmol})$ in EtOH $(5 \mathrm{~mL})$. The reaction mixture was stirred at room temperature until the disappearance of the oxime as shown on TLC. Concentration of the solvent under reduced pressure followed by flash chromatography of the residue afforded the isoxazoline.

General procedure for acetylation of alcohol. To a solution of the alcohol ( $1 \mathrm{mmol})$ in pyridine $(10 \mathrm{~mL})$ was added acetic anhydride $(1.2 \mathrm{mmol})$ and a catalytic amount of DMAP $(0.1 \mathrm{mmol})$ at 0 ${ }^{\circ} \mathrm{C}$. After the addition, the mixture was warmed to room temperature then stirred at the same temperature until the reaction was completed. Concentration of the solvent under reduced pressure followed by flash chromatography afforded the acetate.

Oximolactone 2. Following the general procedure for the formation of oxime (method A), lactol 7 (25.4 mg, $0.136 \mathrm{mmol}$ ) was converted into crude oxime. It was then dissolved in $\mathrm{CH}_{2} \mathrm{Cl}_{2}(4 \mathrm{~mL})$, and $13 \%$ aqueous sodium hypochlorite solution $(0.4 \mathrm{~mL}, 0.699 \mathrm{mmol})$ was added at $0{ }^{\circ} \mathrm{C}$. The mixture was stirred vigorously at the same temperature for $3 \mathrm{~h}$. The reaction mixture was partitioned between $\mathrm{CH}_{2} \mathrm{Cl}_{2}(5 \mathrm{~mL})$ and water $(5 \mathrm{~mL})$. The aqueous layer was extracted with $\mathrm{CH}_{2} \mathrm{Cl}_{2}(3 \times 5$ $\mathrm{mL}$ ) and the combined organic extracts were washed with brine, dried over anhydrous $\mathrm{MgSO}_{4}$, and filtered. Concentration of the filtrate followed by flash chromatography (hexane:EtOAc, 1:1) gave oximolactone $2(21.1 \mathrm{mg}, 78 \%)$ as a white solid: $\mathrm{mp} 152-154{ }^{\circ} \mathrm{C}\left\{\right.$ lit. $^{4}$ enantiomer of 2 had $\mathrm{mp} 153-$ $\left.154{ }^{\circ} \mathrm{C}\right\} ;[\alpha]_{\mathrm{D}}^{20}-36(c 0.66, \mathrm{MeOH})\left\{\right.$ lit. $^{4}$ enantiomer of 2 had $\left.[\alpha]_{\mathrm{D}}^{20}+34(c 0.5, \mathrm{MeOH})\right\} ; \mathrm{R}_{f} 0.2$ (hexane:Et ${ }_{2} \mathrm{O}, 1: 1$ ); IR (thin film) 3420, 2989, 1771, 1687, 1376, 1226, $986 \mathrm{~cm}^{-1} ;{ }^{1} \mathrm{H}$ NMR $\delta 1.38$ $(3 \mathrm{H}, \mathrm{s}), 1.49(3 \mathrm{H}, \mathrm{s}), 4.77-4.84(2 \mathrm{H}, \mathrm{m}), 5.16(1 \mathrm{H}, \mathrm{d}, J=5.7 \mathrm{~Hz}), 5.44(1 \mathrm{H}, \mathrm{d}, J=9.9 \mathrm{~Hz}), 5.50$ $(1 \mathrm{H}, \mathrm{d}, J=17.1 \mathrm{~Hz}), 6.04(1 \mathrm{H}, \mathrm{ddd}, J=17.4,10.2,7.2 \mathrm{~Hz}) ;{ }^{13} \mathrm{C}$ NMR $\delta 26.3\left(\mathrm{CH}_{3}\right), 27.2\left(\mathrm{CH}_{3}\right)$, $78.1(\mathrm{CH}), 79.7(\mathrm{CH}), 85.1(\mathrm{CH}), 114.6(\mathrm{C}), 121.7\left(\mathrm{CH}_{2}\right), 130.9(\mathrm{CH}), 157.8(\mathrm{C})$; MS (CI) $\mathrm{m} / z$ 
(relative intensity) $200\left([\mathrm{MH}]^{+}, 100\right)$; HRMS (CI) calcd for $\mathrm{C}_{9} \mathrm{H}_{13} \mathrm{O}_{4} \mathrm{~N}_{1}[\mathrm{MH}]^{+}$200.0917, found 200.0916.

Oxime 3. Following the general procedure for the formation of oxime (method A) and then purification with flash chromatography (hexane: $\left.\mathrm{Et}_{2} \mathrm{O}, 1: 1\right)$, lactol $34(57.0 \mathrm{mg}, 0.28 \mathrm{mmol})$ was converted into oxime 3 as a mixture of $E$ and $Z$ isomers $(61.5 \mathrm{mg}, 100 \%)$ and as a colorless oil: $[\alpha]_{\mathrm{D}}^{20}$ +0.5 (c 0.98, $\mathrm{CHCl}_{3}$ ); $\mathrm{R}_{f}$ 0.19, 0.09 (hexane: $\mathrm{Et}_{2} \mathrm{O}, 1: 1$ ); IR (thin film) 3387, 2987, 1641, 1382, 1220, $1063 \mathrm{~cm}^{-1}$; ${ }^{1} \mathrm{H}$ NMR (in mixture of geometric isomer in ratio of 1:2) $\delta 1.38(4.5 \mathrm{H}, \mathrm{s}), 1.50(3.0 \mathrm{H}, \mathrm{s})$, $1.52(1.5 \mathrm{H}, \mathrm{s}), 2.19-2.29(1.5 \mathrm{H}, \mathrm{m}), 2.34(1.0 \mathrm{H}$, br s$), 2.43-2.58(1.5 \mathrm{H}, \mathrm{m}), 2.92(0.5 \mathrm{H}, \mathrm{br} \mathrm{s}), 3.66-$ $3.78(1.5 \mathrm{H}, \mathrm{m}), 4.08(1.0 \mathrm{H}, \mathrm{dd}, J=8.4,6.3 \mathrm{~Hz}), 4.20(0.5 \mathrm{H}, \mathrm{dd}, J=7.8,6.3 \mathrm{~Hz}), 4.77(1.0 \mathrm{H}, \mathrm{t}, J=$ $6.3 \mathrm{~Hz}), 5.16(1.5 \mathrm{H}, \mathrm{s}), 5.20(1.5 \mathrm{H}, \mathrm{d}, J=4.8 \mathrm{~Hz}), 5.36(0.5 \mathrm{H}, \mathrm{t}, J=6.3 \mathrm{~Hz}), 5.78-5.92(1.5 \mathrm{H}, \mathrm{m})$, $6.92(0.5 \mathrm{H}, \mathrm{d}, J=5.7 \mathrm{~Hz}), 7.52(1.0 \mathrm{H}, \mathrm{d}, J=7.2 \mathrm{~Hz}), 8.01(1.0 \mathrm{H}$, br s $) ;{ }^{13} \mathrm{C} \mathrm{NMR} \delta 25.7,25.8,28.0$, 28.2, 38.3, 38.9, 69.1, 70.4, 71.8, 75.6, 80.5, 81.2, 110.2, 110.4, 118.4, 119.3, 134.1, 134.6, 150.1, 152.6; MS (ESI) $m / z$ (relative intensity) $238\left([\mathrm{M}+\mathrm{Na}]^{+}, 100\right), 216\left([\mathrm{MH}]^{+}, 20\right), 183(13)$; HRMS (ESI) calcd for $\mathrm{C}_{10} \mathrm{H}_{17} \mathrm{O}_{4} \mathrm{~N}[\mathrm{M}+\mathrm{Na}]^{+} 238.1050$, found 238.1055.

Lactone 4. To a solution of oxime $3(40.1 \mathrm{mg}, 0.19 \mathrm{mmol})$ in $\mathrm{CH}_{2} \mathrm{Cl}_{2}(10 \mathrm{~mL})$ at $0{ }^{\circ} \mathrm{C}$ was added $13 \%$ aqueous sodium hypochlorite solution $(0.89 \mathrm{~mL}, 1.86 \mathrm{mmol})$. The mixture was stirred vigorously at $0{ }^{\circ} \mathrm{C}$ for $3 \mathrm{~h}$. The reaction mixture was partitioned between $\mathrm{CH}_{2} \mathrm{Cl}_{2}(10 \mathrm{~mL})$ and water $(10 \mathrm{~mL})$. The aqueous layer was extracted with $\mathrm{CH}_{2} \mathrm{Cl}_{2}(2 \times 10 \mathrm{~mL})$ and the combined organic extracts were washed with brine, dried over anhydrous $\mathrm{MgSO}_{4}$, and filtered. Concentration of the filtrate followed by flash chromatography (hexane: $\left.\mathrm{Et}_{2} \mathrm{O}, 1: 1\right)$ gave lactone $4(14.0 \mathrm{mg}, 38 \%)$ as a colorless oil: $[\alpha]_{\mathrm{D}}^{20}-66.3\left(c 0.91, \mathrm{CHCl}_{3}\right)\left\{\right.$ lit. $^{6}$ enantiomer of 4 had $[\alpha]_{\mathrm{D}}^{25}+52.5$ (c 0.4., $\left.\left.\mathrm{CHCl}_{3}\right)\right\} ; \mathrm{R}_{f}$ 0.34 (hexane:Et ${ }_{2} \mathrm{O}, 1: 1$ ); IR (thin film) 2990, 1784, 1643, 1378, 1218, $1183 \mathrm{~cm}^{-1}$; ${ }^{1} \mathrm{H}$ NMR $\delta 1.37$ $(3 \mathrm{H}, \mathrm{s}), 1.46(3.0 \mathrm{H}, \mathrm{s}), 2,45(2 \mathrm{H}, \mathrm{t}, J=6 \mathrm{~Hz}), 4.56(1 \mathrm{H}, \mathrm{d}, J=6 \mathrm{~Hz}), 4.65(1 \mathrm{H}, \mathrm{t}, J=6 \mathrm{~Hz}), 4.71$ $(1 \mathrm{H}, \mathrm{d}, J=5.7 \mathrm{~Hz}), 5.21(1 \mathrm{H}, \mathrm{d}, J=3.9 \mathrm{~Hz}), 5.26(1 \mathrm{H}, \mathrm{s}), 5.65-5.79(1 \mathrm{H}, \mathrm{m}) ;{ }^{13} \mathrm{C}$ NMR $\delta 26.1$ $\left(\mathrm{CH}_{3}\right), 27.2\left(\mathrm{CH}_{3}\right), 37.8\left(\mathrm{CH}_{2}\right), 75.4(\mathrm{CH}), 79.1(\mathrm{CH}), 82.3(\mathrm{CH}), 114.2(\mathrm{C}), 121.3\left(\mathrm{CH}_{2}\right), 130.8$ (CH), 174.2 (C); MS (ESI) m/z (relative intensity) 221 ([M+Na $\left.]^{+}, 100\right), 216(10), 199\left([\mathrm{MH}]^{+}, 5\right)$; HRMS (ESI) calcd for $\mathrm{C}_{10} \mathrm{H}_{14} \mathrm{O}_{4}[\mathrm{M}+\mathrm{Na}]^{+} 221.0784$, found 221.0788.

Oxime 5. Following the general procedure for glycol cleavage, the formation of oxime (method $\mathrm{A}$ ), and then purification with flash chromatography (hexane: $\mathrm{Et}_{2} \mathrm{O}, 1: 3$ ), alkene 37 (136 mg, 0.445 mmol) was converted into oxime 5 (126 mg, 98\% overall yield from 37) as a colorless oil: $[\alpha]_{\mathrm{D}}^{20}-$ 183.9 (c 2.10, $\mathrm{CHCl}_{3}$ ); $\mathrm{R}_{f} 0.55$ (hexane:EtOAc, 1:2); IR (thin film) 3390, 2950, 1377, 1124, 1036, 
957, $887 \mathrm{~cm}^{-1} ;{ }^{1} \mathrm{H}$ NMR $(E: Z=1: 0.3) \delta 1.29$ (7.8H, s), 2.16-2.30 (1.3H, m), 2.46-2.59 (1.3H, m), 3.26-3.28 (7.8H, 3s), 3.66-3.78 (2.6H, m), 4.33 (1H, dd, $J=9.3,7.5 \mathrm{~Hz}), 5.02-5.20(2.9 \mathrm{H}, \mathrm{m}), 5.81$ $(1.3 \mathrm{H}, \mathrm{ddt}, J=17.1,9.9,6.9 \mathrm{~Hz}), 6.87(0.3 \mathrm{H}, \mathrm{m}), 7.41(1 \mathrm{H}, \mathrm{d}, J=7.2 \mathrm{~Hz}) ;{ }^{1} \mathrm{H}$ NMR $\left(\mathrm{CDCl}_{3}-\mathrm{D}_{2} \mathrm{O}\right)$ $(E: Z=1: 0.3) \delta 1.29(7.8 \mathrm{H}, \mathrm{s}), 2.16-2.30(1.3 \mathrm{H}, \mathrm{m}), 2.45-2.58(1.3 \mathrm{H}, \mathrm{m}), 3.26-3.28(7.8 \mathrm{H}, 3 \mathrm{~s}), 3.63-$ $3.77(2.6 \mathrm{H}, \mathrm{m}), 4.33(1 \mathrm{H}, \mathrm{dd}, J=9.6,7.5 \mathrm{~Hz}), 5.02-5.16(2.9 \mathrm{H}, \mathrm{m}), 5.81(1.3 \mathrm{H}, \mathrm{ddt}, J=17.1,9.9$, $7.2 \mathrm{~Hz}), 6.88(0.3 \mathrm{H}, \mathrm{d}, J=6.9 \mathrm{~Hz}), 7.41(1 \mathrm{H}, \mathrm{d}, J=7.5 \mathrm{~Hz}) ;{ }^{13} \mathrm{C} \mathrm{NMR} \delta 17.8,17.9,37.2,37.4,48.6$, 48.7, 63.2, 68.9, 70.9, 71.1, 71.6, 71.9, 98.7, 99.0, 99.1, 118.2, 118.6, 134.9, 135.3, 149.2, 149.4; MS (ESI) $\mathrm{m} / z$ (relative intensity) $312\left([\mathrm{M}+\mathrm{Na}]^{+}, 100\right)$; HRMS (ESI) calcd for $\mathrm{C}_{13} \mathrm{H}_{23} \mathrm{O}_{6} \mathrm{~N}[\mathrm{M}+\mathrm{Na}]^{+}$ 312.1418, found 312.1423.

Isoxazoline 6. To a stirred suspension of oxime $5(55.7 \mathrm{mg}, 0.193 \mathrm{mmol})$ and silica gel $(250 \mathrm{mg})$ in EtOH (4 mL) was added chloramine-T trihydrate $(81.5 \mathrm{mg}, 0.290 \mathrm{mmol})$ in small portions. After stirring at room temperature for $5 \mathrm{~min}$, the silica gel was filtered. Concentration of the filtrate followed by flash chromatography (hexane:EtOAc, 2:1 to 1:2) of the residue afforded isoxazoline 6 $(52.0 \mathrm{mg}, 94 \%)$ as a white solid: $\mathrm{mp} 232-233{ }^{\circ} \mathrm{C}$; $\left.[\alpha]_{\mathrm{D}}^{20}-10.9(c) 1.96, \mathrm{CHCl}_{3}\right) ; \mathrm{R}_{f} 0.23$ (hexane:EtOAc, 1:2); IR (thin film) 3545, 2949, 1377, 1118, 1032, $873 \mathrm{~cm}^{-1} ;{ }^{1} \mathrm{H}$ NMR $\delta 1.33(3 \mathrm{H}$, s), $1.37(3 \mathrm{H}, \mathrm{s}), 1.53(1 \mathrm{H}, \mathrm{ddd}, J=14.1,11.7,2.4 \mathrm{~Hz}), 2.32(1 \mathrm{H}, \mathrm{ddd}, J=14.1,6.6,3.3 \mathrm{~Hz}), 3.22$ $(3 \mathrm{H}, \mathrm{s}), 3.30(3 \mathrm{H}, \mathrm{s}), 3.66-3.87$ (3H, $1 \mathrm{td}, J=10.8,6.9 \mathrm{~Hz}, 1 \mathrm{dd}, J=10.2,2.1 \mathrm{~Hz}, 1 \mathrm{dd}, J=10.2,7.8$ $\mathrm{Hz}), 4.15(1 \mathrm{H}, \mathrm{q}, J=2.7 \mathrm{~Hz}), 4.55(1 \mathrm{H}, \mathrm{dd}, J=10.2,7.5 \mathrm{~Hz}), 4.95(1 \mathrm{H}, \mathrm{d}, J=9.9 \mathrm{~Hz}) ;{ }^{13} \mathrm{C} \mathrm{NMR} \delta$ $18.0\left(\mathrm{CH}_{3}\right), 35.0\left(\mathrm{CH}_{2}\right), 44.9(\mathrm{CH}), 48.4\left(\mathrm{CH}_{3}\right), 48.7\left(\mathrm{CH}_{3}\right), 64.6(\mathrm{CH}), 68.8(\mathrm{CH}), 73.9\left(\mathrm{CH}_{2}\right), 74.1$ (CH), 100.7 (C), 100.8 (C), 157.2 (C); MS (ESI) m/z (relative intensity) 310 ([M+Na $\left.]^{+}, 100\right)$; HRMS (ESI) calcd for $\mathrm{C}_{13} \mathrm{H}_{21} \mathrm{O}_{6} \mathrm{~N}[\mathrm{M}+\mathrm{Na}]^{+}$310.1261, found 310.1265; Anal. Calcd for $\mathrm{C}_{13} \mathrm{H}_{21} \mathrm{O}_{6} \mathrm{~N}$ : $\mathrm{C}$, 54.35; H, 7.37; N, 4.87, found: C, 53.57; H, 7.26; N, 4.64.

Isoxazoline 6. Following the general procedure of INOC (method II) and purification with flash chromatography (hexane:EtOAc, 2:1 to $1: 2)$, oxime 5 (88.0 $\mathrm{mg}, 0.304 \mathrm{mmol})$ was converted into isoxazoline $6(53.0 \mathrm{mg}, 61 \%)$ as a white solid.

Lactol 7. To a stirred solution of alkene $39^{13}(302 \mathrm{mg}, 1.04 \mathrm{mmol})$ in dry ether $(10 \mathrm{~mL})$ was added periodic acid $(716 \mathrm{mg}, 3.14 \mathrm{mmol})$ at room temperature under $\mathrm{N}_{2}$. Stirring was continued for $7 \mathrm{~h}$ and the reaction mixture was worked up by filtering and evaporating the filtrate. The residue was purified by flash chromatography (hexane: $\left.\mathrm{Et}_{2} \mathrm{O}, 1: 1\right)$ to give lactol $7(137 \mathrm{mg}, 71 \%$ ) as a colorless oil: $[\alpha]_{\mathrm{D}}^{20}+38.4\left(c\right.$ 1.43, $\mathrm{CHCl}_{3}$ ); $\mathrm{R}_{f} 0.43$ (hexane:Et ${ }_{2} \mathrm{O}, 1: 2$ ); IR (thin film) 3422, 2987, 2940, 1732, 1644, 1376, 1211, 1072, $868 \mathrm{~cm}^{-1}$; ${ }^{1} \mathrm{H}$ NMR $\delta 1.05(3 \mathrm{H}), 1.46(3 \mathrm{H}), 3.37(1 \mathrm{H}, \mathrm{d}, J=2.4$ 
$\mathrm{Hz}), 4.58-4.64(2 \mathrm{H}, \mathrm{m}), 4.71(1 \mathrm{H}, \mathrm{dd}, J=5.7,3.9 \mathrm{~Hz}), 5.31-5.42(3 \mathrm{H}, \mathrm{m}), 5.97(1 \mathrm{H}, \mathrm{ddd}, J=17.5$, 10.35, 7.2 Hz); ${ }^{13} \mathrm{C}$ NMR $\delta 25.3\left(\mathrm{CH}_{3}\right), 26.5\left(\mathrm{CH}_{3}\right), 81.8(\mathrm{CH}), 81.9(\mathrm{CH}), 86.2(\mathrm{CH}), 101.4(\mathrm{CH})$, 113.1 (C), $119.8\left(\mathrm{CH}_{2}\right), 132.5(\mathrm{CH})$; MS (ESI) $\mathrm{m} / z$ (relative intensity) 209 ([M+Na $\left.]^{+}, 100\right)$; HRMS (ESI) calcd for $\mathrm{C}_{9} \mathrm{H}_{14} \mathrm{O}_{4}[\mathrm{M}+\mathrm{Na}]^{+} 209.0784$, found 209.0779.

Isoxazoline 8. Following the general procedure for the formation of oxime (method A), INOC (method I) and then purification with flash chromatography (hexane:EtOAc, 1:1), lactol 7 (137 mg, $0.735 \mathrm{mmol})$ was converted into isoxazoline $8(127 \mathrm{mg}, 87 \%)$ as a white solid: $\mathrm{mp} 109-11{ }^{\circ} \mathrm{C} ;[\alpha]_{\mathrm{D}}^{20}$ -228.2 (c 0.84, $\mathrm{CHCl}_{3}$ ); $\mathrm{R}_{f} 0.4$ (hexane: $\mathrm{Et}_{2} \mathrm{O}, 1: 2$ ); IR (thin film) 3417, 2989, 2939, 1646, 1503, 1378, 1210, 1054, $869 \mathrm{~cm}^{-1} ;{ }^{1} \mathrm{H}$ NMR $\left(\mathrm{C}_{6} \mathrm{D}_{6}\right) \delta 1.05$ (3H, s), 1.35 (3H, s), 2.04 (1H, brs), 3.58-3.65 $(2 \mathrm{H}, \mathrm{m}), 3.96(1 \mathrm{H}, \mathrm{dd}, J=11.4,8.1 \mathrm{~Hz}), 4.11(1 \mathrm{H}, \mathrm{t}, J=9.3 \mathrm{~Hz}), 4.41(1 \mathrm{H}, \mathrm{d}, J=5.1 \mathrm{~Hz}), 4.89$ $(1 \mathrm{H}, \mathrm{d}, J=5.1 \mathrm{~Hz}) ;{ }^{13} \mathrm{C}$ NMR $\delta 24.2\left(\mathrm{CH}_{3}\right), 26.5\left(\mathrm{CH}_{3}\right), 55.1(\mathrm{CH}), 69.3\left(\mathrm{CH}_{2}\right), 69.9(\mathrm{CH}), 71.4$ $(\mathrm{CH}), 90.6(\mathrm{CH}), 112.9(\mathrm{C}), 164.7(\mathrm{C})$; MS (ESI) $\mathrm{m} / z$ (relative intensity) 222 ([M+Na $\left.]^{+}, 100\right), 200$ (4); HRMS (ESI) calcd for $\mathrm{C}_{9} \mathrm{H}_{13} \mathrm{O}_{4} \mathrm{~N}_{1}[\mathrm{M}+\mathrm{Na}]^{+} 222.0737$, found 222.0740.

Isoxazoline 8. Following the general procedure for the formation of oxime (method A), INOC (method II) and purification with flash chromatography (hexane:Et ${ }_{2} \mathrm{O}, 1: 2$ to hexane:EtOAc, 1:1), lactol 7 (27.8 $\mathrm{mg}, 0.149 \mathrm{mmol})$ was converted into isoxazoline $8(15.7 \mathrm{mg}, 53 \%)$ and oximolactone 2 (9.8 $\mathrm{mg}, 33 \%)$ as white solids.

Acetylated isoxazoline 8a. Following the general procedure for the acetylation of alcohol and then purification with flash chromatography (hexane: $\left.\mathrm{Et}_{2} \mathrm{O}, 1: 1\right)$, isoxazoline 8 (26 $\left.\mathrm{mg}, 0.13 \mathrm{mmol}\right)$ was acetylated to afford acetate $8 \mathbf{a}(31 \mathrm{mg}, 99 \%)$ as a white solid: $\mathrm{mp} 164-166{ }^{\circ} \mathrm{C}$ \{ lit. $^{8}$ the enantiomer of isoxazoline 8a had mp $\left.163{ }^{\circ} \mathrm{C}\right\} ;[\alpha]_{\mathrm{D}}^{20}-152\left(c\right.$ 1.63, MeOH) $\left\{\right.$ lit. ${ }^{8}$ the enantiomer of isoxazoline 8a had $[\alpha]_{\mathrm{D}}^{20}+154(c$ 0.5, MeOH) $\} ; \mathrm{R}_{f} 0.5$ (hexane:EtOAc, 1:1); IR (thin film) 2991, 2964, 1738, 1647, 1377, 1237, $860 \mathrm{~cm}^{-1}$; ${ }^{1} \mathrm{H}$ NMR $\delta 1.34(3 \mathrm{H}, \mathrm{s}), 1.50(3 \mathrm{H}, \mathrm{s}), 2.08(3 \mathrm{H}, \mathrm{s}), 4.01(1 \mathrm{H}, \mathrm{t}, J=9 \mathrm{~Hz})$, $4.24(1 \mathrm{H}, \mathrm{dt}, J=9.9,5.4 \mathrm{~Hz}), 4.46(1 \mathrm{H}, \mathrm{dd}, J=11.4,8.7 \mathrm{~Hz}), 4.80(1 \mathrm{H}, \mathrm{d}, J=5.1 \mathrm{~Hz}), 5.07(1 \mathrm{H}, \mathrm{d}$, $J=5.4 \mathrm{~Hz}), 5.10(1 \mathrm{H}, \mathrm{d}, J=5.4 \mathrm{~Hz}) ;{ }^{13} \mathrm{C} \mathrm{NMR} \delta 21.1\left(\mathrm{CH}_{3}\right), 24.2\left(\mathrm{CH}_{3}\right), 26.5\left(\mathrm{CH}_{3}\right), 53.3(\mathrm{CH})$, $69.7\left(\mathrm{CH}_{2}\right), 71.5(\mathrm{CH}), 72.1(\mathrm{CH}), 88.6(\mathrm{CH}), 113.3(\mathrm{C}), 163.6(\mathrm{C}), 170.1(\mathrm{C})$; MS (CI) $\mathrm{m} / z$ (relative intensity) $242\left([\mathrm{MH}]^{+}, 100\right), 184$ (41), 182 (11), 124 (4); HRMS (CI) calcd for $\mathrm{C}_{11} \mathrm{H}_{15} \mathrm{~N}_{1} \mathrm{O}_{5}[\mathrm{MH}]^{+}$ 242.1023 , found 242.1025 . 
Oxime 9: To a solution of iodide $41^{14}(221.1 \mathrm{mg}, 0.7271 \mathrm{mmol})$ in $\mathrm{THF} / \mathrm{H}_{2} \mathrm{O}(\mathrm{v} / \mathrm{v}=4: 1)(15 \mathrm{~mL})$ was added activated $\mathrm{Zn}$ powder $(500 \mathrm{mg}, 7.6475 \mathrm{mmol})$ at room temperature. The reaction mixture was sonicated at $40{ }^{\circ} \mathrm{C}$ for $45 \mathrm{~min}$. The $\mathrm{Zn}$ powder was filtered and the filtrate was concentrated to afford crude lactol 42. Following the oxime formation procedure (method B) and purification with flash chromatography (EtOAc:MeOH, 20:1), oxime 9 (109 mg, 93\% overall yield from 41) was obtained as a colorless syrup: $[\alpha]_{\mathrm{D}}^{20} 11.5^{\circ}(c 1.33 \mathrm{MeOH}) ; \mathrm{R}_{f} 1.5\left(\mathrm{CHCl}_{3}: \mathrm{MeOH}, 9: 1\right)$; ${ }^{1} \mathrm{H}$ NMR $(E: Z$ $=2: 1)\left(\mathrm{CD}_{3} \mathrm{OD}\right) \delta 3.45(1 \mathrm{H}, \mathrm{dd}, J=5.4,3.9 \mathrm{~Hz}), 3.64(0.4 \mathrm{H}, \mathrm{dd}, J=6.8,2.6 \mathrm{~Hz}), 4.19(1.5 \mathrm{H}, \mathrm{dd}, J$ $=6.6,6.0 \mathrm{~Hz}), 4.25(1.2 \mathrm{H}, \mathrm{dd}, J=6.9,4.2 \mathrm{~Hz}), 5.19(1.6 \mathrm{H}, \mathrm{ddd}, J=10.5,1.87,1.5 \mathrm{~Hz}$ and ddd, $J=$ $10.5,1.8,1.2 \mathrm{~Hz}), 5.34(1.7 \mathrm{H}, \mathrm{dt}, J=17.1, \mathrm{~Hz}$ and dt, $J=17.4,1.5 \mathrm{~Hz}), 5.94(1.5 \mathrm{H}, \mathrm{ddd}, J=17.1$, $10.5,6.6 \mathrm{~Hz}$ and ddd, $J=17.4,10.2,6.6 \mathrm{~Hz}), 6.76(0.3 \mathrm{H}, \mathrm{d}, J=3.7 \mathrm{~Hz}), 7.44(1.0 \mathrm{H}, \mathrm{d}, J=6.9 \mathrm{~Hz})$; ${ }^{13} \mathrm{C}$ NMR $\left(\mathrm{CD}_{3} \mathrm{OD}\right) \delta 66.6(\mathrm{CH}), 70.9(\mathrm{CH}), 74.3(\mathrm{CH}), 75.0(\mathrm{CH}), 75.9(\mathrm{CH}), 77.1(\mathrm{CH}), 117.0$ $\left(\mathrm{CH}_{2}\right), 117.3\left(\mathrm{CH}_{2}\right), 138.9(\mathrm{CH}), 149.2(\mathrm{CH}), 151.8(\mathrm{CH}), 154.0(\mathrm{CH}) ; \mathrm{MS}(\mathrm{ESI}) \mathrm{m} / z$ (relative intensity) $184\left([\mathrm{M}+\mathrm{Na}]^{+}, 100\right), 162\left([\mathrm{MH}]^{+}, 25\right)$; HRMS (ESI) calcd for $\mathrm{C}_{10} \mathrm{H}_{17} \mathrm{O}_{4} \mathrm{~N}[\mathrm{M}+\mathrm{Na}]^{+}$ 184.0580, found 184.0587 .

Isoxazoline 10. Following the INOC procedure (method I) and purification with flash chromatography $\left(\mathrm{CHCl}_{3}\right.$ to $\left.\mathrm{CHCl}_{3}: \mathrm{MeOH}, 10: 1\right)$, oxime 9 (46.0 mg, 0.289mmol) was converted into isoxazoline $10(40 \mathrm{mg}, 87 \%)$ as a colorless oil: $[\alpha]_{\mathrm{D}}^{20}-113.1^{\circ}(c \quad 0.495, \mathrm{MeOH}) ; \mathrm{R}_{f} 0.1$ $\left(\mathrm{CHCl}_{3}: \mathrm{MeOH}, 9: 1\right) ;{ }^{1} \mathrm{H}$ NMR $\left(\mathrm{CD}_{3} \mathrm{OD}\right) \delta 3.57(1 \mathrm{H}, \mathrm{dd}, J=8.4,7.2), 3.80(1 \mathrm{H}, \mathrm{ddt}, J=11.4,10.2$, $8.7 \mathrm{~Hz}), 3.98(1 \mathrm{H}, \mathrm{dd}, J=11.4,8.1 \mathrm{~Hz}), 4.06(1 \mathrm{H}, \mathrm{dd}, J=7.2,5.1 \mathrm{~Hz}), 4.30(1 \mathrm{H}, \mathrm{dd}, J=5.1,1.5$ $\mathrm{Hz}), 4.62(1 \mathrm{H}, \mathrm{dd}, J=9.9,8.1 \mathrm{~Hz}) ;{ }^{13} \mathrm{C} \mathrm{NMR}\left(\mathrm{CD}_{3} \mathrm{OD}\right) \delta 59.0(\mathrm{CH}), 72.4(\mathrm{CH}), 75.9\left(\mathrm{CH}_{2}\right), 80.2$ $(\mathrm{CH}), 90.7(\mathrm{CH}), 165.3(\mathrm{C})$; MS (ESI) $\mathrm{m} / z$ (relative intensity) $182\left([\mathrm{M}+\mathrm{Na}]^{+}, 100\right), 216\left([\mathrm{MH}]^{+}, 19\right)$; HRMS (ESI) calcd for $\mathrm{C}_{6} \mathrm{H}_{9} \mathrm{O}_{4} \mathrm{~N}[\mathrm{M}+\mathrm{Na}]^{+} 182.0424$, found 182.0432 .

Isoxazoline 10. Following the INOC procedure (method II) and purification with flash chromatography $\left(\mathrm{CHCl}_{3}\right.$ to $\left.\mathrm{CHCl}_{3}: \mathrm{MeOH}, 10: 1\right)$, oxime 9 (18.0 mg, $\left.0.111 \mathrm{mmol}\right)$ was converted into isoxazoline $\mathbf{1 0}(9 \mathrm{mg}, 51 \%)$ as a colorless oil.

Acetylated isoxazoline 10a. Following the general procedure for the acetylation of alcohol and purification with flash chromatography (hexane:EtOAc, 2:1), isoxazoline 10 (34.3 $\mathrm{mg}, 0.4103$ mmol) was acetylated to afford isoxazoline 10a (116 mg, 99\%) as a white solid: $\mathrm{mp} 111-112{ }^{\circ} \mathrm{C}$ $\left\{\right.$ lit. $\left.{ }^{9} \mathrm{mp} 111-112^{\circ} \mathrm{C}\right\} ;[\alpha]_{\mathrm{D}}^{20}-102.5^{\circ}\left(c 0.98, \mathrm{CHCl}_{3}\right)\left\{\right.$ lit. $\left.^{9}[\alpha]_{\mathrm{D}}^{20}-101^{\circ}\left(c \quad 1.07, \mathrm{CHCl}_{3}\right)\right\} ; \mathrm{R}_{f} 0.38$ (hexane:EtOAc, 1:1); IR (thin film) 3474, 3020, 2960, 1745, 1635, 1376, 1200, 1169, 1120, 1032 $\mathrm{cm}^{-1} ;{ }^{1} \mathrm{H}$ NMR $\delta 2.10(3 \mathrm{H}, \mathrm{s}), 2.12(3 \mathrm{H}, \mathrm{s}), 2.13(3 \mathrm{H}, \mathrm{s}), 3.90(1 \mathrm{H}, \mathrm{dddd}, J=11.9,10.3,8.3,1.3 \mathrm{~Hz})$, 
$4.34(1 \mathrm{H}, \mathrm{dd}, J=9.3,6.0 \mathrm{~Hz}), 4.68(1 \mathrm{H}, \mathrm{dd}, J=8.4,6.9), 4.73(1 \mathrm{H}, \mathrm{dd}, J=10.2,9.3 \mathrm{~Hz}), 5.53(1 \mathrm{H}$, $\mathrm{dd}, J=3.9,1.5 \mathrm{~Hz}), 5.66(1 \mathrm{H}, \mathrm{dd}, J=6.6,3.9 \mathrm{~Hz}){ }^{13} \mathrm{C} \mathrm{NMR} \delta 21.2\left(\mathrm{CH}_{3}\right), 21.2\left(\mathrm{CH}_{3}\right), 21.2\left(\mathrm{CH}_{3}\right)$, $56.2(\mathrm{CH}), 69.7(\mathrm{CH}), 76.3\left(\mathrm{CH}_{2}\right), 84.1(\mathrm{CH}), 159.0(\mathrm{C}), 169.9(\mathrm{C}), 170.0(\mathrm{C}), 171.2(\mathrm{C})$; MS (ESI) $m / z$ (relative intensity) $286\left([\mathrm{MH}]^{+}, 100\right), 226(86)$; HRMS (CI) calcd for $\mathrm{C}_{12} \mathrm{H}_{15} \mathrm{O}_{7} \mathrm{~N}[\mathrm{M}+\mathrm{H}]^{+}$ 286.0921, found 286.0928 .

Isoxazolines 11 and 12 from oxime 3. Following the general procedure for the INOC reaction (method I) and purification with flash chromatography (hexane:EtOAc, 1:2 to EtOAc), oxime 3 (119 $\mathrm{mg}, 0.55 \mathrm{mmol}$ ) was converted into isoxazoline 11 (76.6 $\mathrm{mg}, 65 \%$ from 34) and isoxazoline 12 (16.4 $\mathrm{mg}, 14 \%$ from 34) as colorless oils. Data for 11: $[\alpha]_{\mathrm{D}}^{20}-79.2$ (c 3.84, $\left.\mathrm{CHCl}_{3}\right) ; \mathrm{R}_{f} 0.28$ (hexane:EtOAc, 1:2); IR (thin film) 3436, 2988, 2938, 1630, 1451, 1382, 1211, $1046 \mathrm{~cm}^{-1}$; ${ }^{1} \mathrm{H}$ NMR $\delta 1.43(3 \mathrm{H}, \mathrm{s}), 1.56(3 \mathrm{H}, \mathrm{s}), 1.58-1.63(1 \mathrm{H}, \mathrm{m}), 2.29(1 \mathrm{H}, \mathrm{ddd}, J=13.5,9,7.8 \mathrm{~Hz}), 2.31$ (1H, br s), 3.68-3.82 (2H, m), $3.92(1 \mathrm{H}, \mathrm{dd}, J=10.8,8.1 \mathrm{~Hz}), 4.46(1 \mathrm{H}, \mathrm{dd}, J=7.5,3.6 \mathrm{~Hz}), 4.66(1 \mathrm{H}, \mathrm{dd}, J=$ 10.8, 8.1 Hz), $5.05(1 \mathrm{H}, \mathrm{d}, J=7.5 \mathrm{~Hz}) ;{ }^{13} \mathrm{C} \mathrm{NMR} \delta 24.6\left(\mathrm{CH}_{3}\right), 26.2\left(\mathrm{CH}_{3}\right), 31.0\left(\mathrm{CH}_{2}\right), 40.2(\mathrm{CH})$, $66.2(\mathrm{CH}), 69.3(\mathrm{CH}), 75.9\left(\mathrm{CH}_{2}\right), 77.8(\mathrm{CH}), 111.6(\mathrm{C}), 157.5(\mathrm{C})$; MS (ESI) $m / z$ (relative intensity) $236\left([\mathrm{M}+\mathrm{Na}]^{+}, 100\right), 214\left([\mathrm{MH}]^{+}, 17\right)$; HRMS (ESI) calcd for $\mathrm{C}_{10} \mathrm{H}_{15} \mathrm{O}_{4} \mathrm{~N}[\mathrm{M}+\mathrm{Na}]^{+} 236.0893$, found 236.0897 .

Data for 12: $[\alpha]_{\mathrm{D}}^{20}+78.2\left(c 0.78, \mathrm{CHCl}_{3}\right)$; $\mathrm{R}_{f} 0.09$ (hexane:EtOAc, 1:2); IR (thin film) 3422, 2985, 2933, 1631, 1454, 1383, 1224, $1051 \mathrm{~cm}^{-1} ;{ }^{1} \mathrm{H}$ NMR $\delta 1.42(3 \mathrm{H}, \mathrm{s}), 1.54(3 \mathrm{H}, \mathrm{s}), 1.85(1 \mathrm{H}, \mathrm{q}, J=$ $11.4 \mathrm{~Hz}), 2.12(1 \mathrm{H}, \mathrm{dt}, J=12.3,5.1 \mathrm{~Hz}), 2.12(1 \mathrm{H}, \mathrm{br} \mathrm{s}), 3.26(1 \mathrm{H}, \mathrm{qd}, J=9.9,5.7 \mathrm{~Hz}), 3.98(1 \mathrm{H}$, dd, $J=9.9,8.1 \mathrm{~Hz}), 4.04(1 \mathrm{H}, \mathrm{dt}, J=11.1,3.6 \mathrm{~Hz}), 4.47(1 \mathrm{H}, \mathrm{dd}, J=5.4,3.3 \mathrm{~Hz}), 4.55(1 \mathrm{H}, \mathrm{dd}, J=$ 10.8, 8.1 Hz), $4.89(1 \mathrm{H}, \mathrm{d}, J=5.1 \mathrm{~Hz}) ;{ }^{13} \mathrm{C} \mathrm{NMR} \delta 26.7\left(\mathrm{CH}_{3}\right), 27.9\left(\mathrm{CH}_{3}\right), 33.2\left(\mathrm{CH}_{2}\right), 45.8(\mathrm{CH})$, $68.4(\mathrm{CH}), 71.5(\mathrm{CH}), 74.1\left(\mathrm{CH}_{2}\right), 78.1(\mathrm{CH}), 111.7(\mathrm{C}), 158.7(\mathrm{C})$; MS (ESI) $m / z$ (relative intensity) $236\left([\mathrm{M}+\mathrm{Na}]^{+}, 100\right), 214\left([\mathrm{MH}]^{+}, 19\right)$; HRMS (ESI) calcd for $\mathrm{C}_{10} \mathrm{H}_{15} \mathrm{O}_{4} \mathrm{~N}[\mathrm{M}+\mathrm{Na}]^{+} 236.0893$, found 236.0893 .

Lactol 13. Following the selective hydrolysis-glycol cleavage oxidation procedure for the formation of lactol 7 and purification with flash chromatography (hexane:Et ${ }_{2} \mathrm{O}, 1: 1$ ), alkene $\mathbf{4 0}^{13}$ (401 mg, $1.39 \mathrm{mmol})$ was converted into lactol $13(176 \mathrm{mg}, 68 \%)$ as a colorless oil: $[\alpha]_{\mathrm{D}}^{20}+4.27(c$ 0.74, $\mathrm{CHCl}_{3}$ ); $\mathrm{R}_{f} 0.5$ (hexane: $\mathrm{Et}_{2} \mathrm{O}, 1: 2$ ); IR (thin film) 3423, 2987, 2940, 1731, 1644, 1375, 1211, $1071,867 \mathrm{~cm}^{-1}$; ${ }^{1} \mathrm{H}$ NMR (mixture of $\alpha$ and $\beta$-D-isomers with ratio $\left.\alpha: \beta=4.4: 1\right) \delta 1.33(3 \mathrm{H}, \mathrm{s}), 1.39$ $(0.57 \mathrm{H}, \mathrm{s}), 1.50(3 \mathrm{H}, \mathrm{s}), 1.58(0.53 \mathrm{H}, \mathrm{s}), 2.84(1 \mathrm{H}, \mathrm{d}, J=3 \mathrm{~Hz}), 3.94(0.19 \mathrm{H}, \mathrm{d}, J=10.5 \mathrm{~Hz}), 4.59-$ $4.70(3.23 \mathrm{H}, \mathrm{m}), 5.17(1 \mathrm{H}, \mathrm{dt}, J=10.2,1.2 \mathrm{~Hz}), 5.22(0.25 \mathrm{H}, \mathrm{dt}, J=10.8,1.5 \mathrm{~Hz}), 5.26-5.33$ $(1.15 \mathrm{H}, \mathrm{m}), 5.39(0.29 \mathrm{H}, \mathrm{dt}, J=17.1,1.5 \mathrm{~Hz}), 5.49(1 \mathrm{H}, \mathrm{d}, J=2.7 \mathrm{~Hz}), 5.78(0.23 \mathrm{H}, \mathrm{ddd}, J=17.4$, 
10.5, $4.8 \mathrm{~Hz}), 6.00(1 \mathrm{H}$, ddd, $J=17.1,10.5,7.8 \mathrm{~Hz}) ;{ }^{13} \mathrm{C} \mathrm{NMR} \delta 25.5\left(\mathrm{CH}_{3}\right), 26.7\left(\mathrm{CH}_{3}\right), 26.9$ $\left(\mathrm{CH}_{3}\right), 79.6(\mathrm{CH}), 81.1(\mathrm{CH}), 84.3(\mathrm{CH}), 85.2(\mathrm{CH}), 86.6(\mathrm{CH}), 89.1(\mathrm{CH}), 96.7(\mathrm{CH}), 103.6(\mathrm{CH})$, $112.9(\mathrm{C}), 114.8(\mathrm{C}), 117.5\left(\mathrm{CH}_{2}\right), 117.8\left(\mathrm{CH}_{2}\right), 135.0(\mathrm{CH}), 138.5(\mathrm{CH})$; MS (ESI) $m / z$ (relative intensity) 237 (100), 209 ([M+Na $\left.]^{+}, 11\right)$; HRMS (ESI) calcd for $\mathrm{C}_{9} \mathrm{H}_{14} \mathrm{O}_{4}[\mathrm{M}+\mathrm{Na}]^{+} 209.0784$, found 209.0789 .

Isoxazoline 14. According to the general procedure for the formation of oxime (method A), INOC reaction (method I) and purification with flash chromatography (hexane:EtOAc, 1:1), lactol 13 (110 $\mathrm{mg}, 0.59 \mathrm{mmol})$ was converted into isoxazoline $14(108 \mathrm{mg}, 92 \%)$ as a colorless oil: $[\alpha]_{\mathrm{D}}^{20}-95.4(c$ 0.85, $\mathrm{CHCl}_{3}$ ); $\mathrm{R}_{f} 0.16$ (hexane:EtOAc, 1:1); IR (thin film) 3417, 2988, 2938, 1645, 1502, 1378, 1054, $868 \mathrm{~cm}^{-1}$; ${ }^{1} \mathrm{H}$ NMR $\delta 1.38(3 \mathrm{H}, \mathrm{s}), 1.54(3 \mathrm{H}, \mathrm{s}), 2.88(1 \mathrm{H}, \mathrm{d}, J=10.2 \mathrm{~Hz}), 3.74(1 \mathrm{H}, \mathrm{dt}, J=$ 9.3, $5.4 \mathrm{~Hz}), 3.88(1 \mathrm{H}, \mathrm{q}, J=9.3 \mathrm{~Hz}), 4.19(1 \mathrm{H}, \mathrm{t}, J=9 \mathrm{~Hz}), 4.62(1 \mathrm{H}, \mathrm{dd}, J=10.5,8.7 \mathrm{~Hz}), 4.85$ $(1 \mathrm{H}, \mathrm{t}, J=5.4 \mathrm{~Hz}), 5.01(1 \mathrm{H}, \mathrm{d}, J=5.4 \mathrm{~Hz}) ;{ }^{13} \mathrm{C} \mathrm{NMR} \delta 24.4\left(\mathrm{CH}_{3}\right), 26.3\left(\mathrm{CH}_{3}\right), 56.9(\mathrm{CH}), 72.3$ $(\mathrm{CH}), 74.4\left(\mathrm{CH}_{2}\right), 74.6(\mathrm{CH}), 83.4(\mathrm{CH}), 113.4(\mathrm{C}), 161.2(\mathrm{C})$; MS (CI) $\mathrm{m} / z$ (relative intensity) 200 $\left([\mathrm{MH}]^{+}, 100\right), 142$ (10); HRMS (CI) calcd for $\mathrm{C}_{9} \mathrm{H}_{13} \mathrm{~N}_{1} \mathrm{O}_{4}[\mathrm{MH}]^{+}$200.0917, found 200.0916.

Acetylated isoxazoline 14a. Following the general procedure for the acetylation of alcohol and purification with flash chromatography (hexane: $\left.\mathrm{Et}_{2} \mathrm{O}, 1: 1\right)$, isoxazoline 14 (11.1 $\left.\mathrm{mg}, 0.055 \mathrm{mmol}\right)$ was acetylated to afford isoxazoline 14a $(13.2 \mathrm{mg}, 99 \%)$ as a white solid. The white solid was recrystallized from $\mathrm{Et}_{2} \mathrm{O}(\sim 1 \mathrm{~mL})$ with hexane $(\sim 2 \mathrm{~mL})$ as colorless crystals: $\mathrm{mp} 101-103{ }^{\circ} \mathrm{C}$; $[\alpha]_{\mathrm{D}}^{20}-$ 85.9 (c $\left.0.91, \mathrm{CHCl}_{3}\right) ; \mathrm{R}_{f} 0.2\left(\mathrm{Et}_{2} \mathrm{O}\right)$; IR (thin film) 2983, 2922, 1739, 1637, 1382, 1235, 1048, 869 $\mathrm{cm}^{-1} ;{ }^{1} \mathrm{H}$ NMR $\delta 1.38(3 \mathrm{H}, \mathrm{s}), 1.55(3 \mathrm{H}, \mathrm{s}), 2.14(3 \mathrm{H}, \mathrm{s}), 4.13(1 \mathrm{H}, \mathrm{q}, J=9.9 \mathrm{~Hz}), 4.28(1 \mathrm{H}, \mathrm{dd}, J=$ $10.5,8.7 \mathrm{~Hz}), 4.54(1 \mathrm{H}, \mathrm{dd}, J=9.3,4.5 \mathrm{~Hz}), 4.63(1 \mathrm{H}, \mathrm{dd}, J=10.5,8.7 \mathrm{~Hz}), 5.03(1 \mathrm{H}, \mathrm{d}, J=5.4$ $\mathrm{Hz}), 5.09(1 \mathrm{H}, \mathrm{t}, J=4.8 \mathrm{~Hz}) ;{ }^{13} \mathrm{C} \mathrm{NMR} \delta 21.2\left(\mathrm{CH}_{3}\right), 24.5\left(\mathrm{CH}_{3}\right), 26.6\left(\mathrm{CH}_{3}\right), 54.4(\mathrm{CH}), 72.0(\mathrm{CH})$, $75.0\left(\mathrm{CH}_{2}\right), 75.1(\mathrm{CH}), 83.3(\mathrm{CH}), 113.7(\mathrm{C}), 161.1(\mathrm{C}), 171.1(\mathrm{C})$; MS (ESI) $m / z$ (relative intensity) $264\left([\mathrm{M}+\mathrm{Na}]^{+}, 100\right), 242(8), 182$ (16); HRMS (ESI) calcd for $\mathrm{C}_{11} \mathrm{H}_{15} \mathrm{~N}_{1} \mathrm{O}_{5}[\mathrm{M}+\mathrm{Na}]^{+} 264.0842$, found 264.0843 .

Isoxazoline 16. Following the general procedure for the glycol oxidative cleavage, formation of oxime (method A), INOC reaction (method I), and purification with flash chromatography (hexane:EtOAc, $1: 1)$, alkene $\mathbf{1 5}^{13}(66.9 \mathrm{mg}, 0.310 \mathrm{mmol})$ was converted into isoxazoline 16 (44.4 $\mathrm{mg}, 73 \%$ overall yield from 15) as a colorless oil: $[\alpha]_{\mathrm{D}}^{20} 95.2^{\circ}$ (c 1.89, $\mathrm{CHCl}_{3}$ ); $\mathrm{R}_{f} 0.16$ (hexane:EtOAc, 1:1); IR (thin film) 3445, 2989, 2937, 2350, 1646, 1376, 1267, 1210, 1157, 1102, 1054, $\mathrm{cm}^{-1} ;{ }^{1} \mathrm{H}$ NMR $\delta 1.40(3 \mathrm{H}, \mathrm{s}), 1.55(3 \mathrm{H}, \mathrm{s}), 2.50(1 \mathrm{H}, \mathrm{br} \mathrm{s}), 3.75(1 \mathrm{H}, \mathrm{dd}, J=8.7,5.1 \mathrm{~Hz}), 3.87$ 
$(1 \mathrm{H}, \mathrm{q}, J=9.0), 4.21(1 \mathrm{H}, \mathrm{t}, J=9.0), 4.64(1 \mathrm{H}, \mathrm{dd}, J=10.8,9.0 \mathrm{~Hz}), 4.86(1 \mathrm{H}, \mathrm{t}, J=5.1 \mathrm{~Hz}), 5.03$ $(1 \mathrm{H}, \mathrm{d}, J=5.4 \mathrm{~Hz}) ;{ }^{13} \mathrm{C}$ NMR $\delta 24.5\left(\mathrm{CH}_{3}\right), 26.4\left(\mathrm{CH}_{3}\right), 56.9(\mathrm{CH}), 72.3(\mathrm{CH}), 74.4\left(\mathrm{CH}_{2}\right), 74.6$ $(\mathrm{CH}), 83.6\left(\mathrm{CH}_{3}\right), 113.4(\mathrm{C}), 161.3(\mathrm{C})$; MS (ESI) $\mathrm{m} / \mathrm{z}$ (relative intensity) $222\left([\mathrm{M}+\mathrm{Na}]^{+}, 100\right), 200$ $\left([\mathrm{MH}]^{+}, 42\right)$; HRMS (ESI) calcd for $\mathrm{C}_{9} \mathrm{H}_{13} \mathrm{O}_{4} \mathrm{~N}[\mathrm{M}+\mathrm{Na}]^{+}$222.0737, found 222.0740.

Isoxazolines 18 and 19. Following the general procedure for oxime formation (method A), INOC reaction (method I) and purification with flash chromatography $\left(\mathrm{Et}_{2} \mathrm{O}\right.$ to EtOAc), lactol $17^{16}$ (128.0 $\mathrm{mg}, 0.64 \mathrm{mmol})$ was converted into isoxazoline $18(98.3 \mathrm{mg}, 72 \%$ overall yield from 17) and isoxazoline $19\left(20.0 \mathrm{mg}, 15 \%\right.$ overall yield from 17) as colorless oils. Data for 18: $[\alpha]_{\mathrm{D}}^{20} 78.5^{\circ}(c$ 1.82, $\mathrm{CHCl}_{3}$ ); $\mathrm{R}_{f} 0.28$ (hexane:EtOAc, 1:2); IR (thin film) 3433, 2988, 2937, 1630, 1451, 1382, 1268, 1252, 1211, 1160, 1123, $1046 \mathrm{~cm}^{-1} ;{ }^{1} \mathrm{H}$ NMR $\delta 1.42(3 \mathrm{H}, \mathrm{s}), 1.56(3 \mathrm{H}, \mathrm{s}), 1.58-1.63(1 \mathrm{H}, \mathrm{m})$, $2.29(1 \mathrm{H}, \mathrm{ddd}, J=13.5,9,7.5 \mathrm{~Hz}), 3.68-3.84(2 \mathrm{H}, \mathrm{m}), 3.91(1 \mathrm{H}, \mathrm{dd}, J=11.4,8.1 \mathrm{~Hz}), 4.46(1 \mathrm{H}$, $\mathrm{dd}, J=7.5,3.6 \mathrm{~Hz}), 4.53(1 \mathrm{H}, \mathrm{dd}, J=10.8,8.1 \mathrm{~Hz}), 5.46(1 \mathrm{H}, \mathrm{d}, J=7.5 \mathrm{~Hz}) ;{ }^{13} \mathrm{C}$ NMR $\delta 24.7$ $\left(\mathrm{CH}_{3}\right), 26.4\left(\mathrm{CH}_{3}\right), 31.3\left(\mathrm{CH}_{2}\right), 40.2(\mathrm{CH}), 66.3(\mathrm{CH}), 69.5(\mathrm{CH}), 75.9\left(\mathrm{CH}_{2}\right), 77.9(\mathrm{CH}), 111.7(\mathrm{C})$, 157.5 (C); MS (ESI) $m / z$ (relative intensity) $236\left([\mathrm{M}+\mathrm{Na}]^{+}, 100\right), 214\left([\mathrm{MH}]^{+}, 6\right)$; HRMS (ESI) calcd for $\mathrm{C}_{10} \mathrm{H}_{15} \mathrm{O}_{4} \mathrm{~N}[\mathrm{M}+\mathrm{Na}]^{+} 236.0893$, found 236.0893.

Data for 19: $[\alpha]_{\mathrm{D}}^{20}-75.3^{\circ}\left(c 0.83, \mathrm{CHCl}_{3}\right)$; $\mathrm{R}_{f} 0.09$ (hexane:EtOAc, 1:2); IR (thin film) 3422, 2985, 2933, 1631, 1454, 1383, 1224, $1051 \mathrm{~cm}^{-1} ;{ }^{1} \mathrm{H}$ NMR $\delta 1.34(3 \mathrm{H}, \mathrm{s}), 1.55(3 \mathrm{H}, \mathrm{s}), 1.85(1 \mathrm{H}, \mathrm{q}, J=$ $11.4 \mathrm{~Hz}), 2.13(1 \mathrm{H}, \mathrm{dt}, J=12.3,5.0 \mathrm{~Hz}), 2.28(1 \mathrm{H}, \mathrm{br} \mathrm{s}), 3.27(1 \mathrm{H}, \mathrm{qdd}, J=10.2,5.7,1.2 \mathrm{~Hz}), 3.98$ $(1 \mathrm{H}, \mathrm{dd}, J=9.9,8.1 \mathrm{~Hz}), 4.05(1 \mathrm{H}, \mathrm{m}), 4.48(1 \mathrm{H}, \mathrm{dd}, J=5.1,3.3 \mathrm{~Hz}), 4.52(1 \mathrm{H}, \mathrm{dd}, J=10.8,8.1$ $\mathrm{Hz}), 4.90(1 \mathrm{H}, \mathrm{dd}, J=5.4,1.2 \mathrm{~Hz}) ;{ }^{13} \mathrm{C} \mathrm{NMR} \delta 26.8\left(\mathrm{CH}_{3}\right), 28.0\left(\mathrm{CH}_{3}\right), 33.3\left(\mathrm{CH}_{2}\right), 45.9(\mathrm{CH}), 68.5$ $(\mathrm{CH}), 71.6(\mathrm{CH}), 74.2\left(\mathrm{CH}_{2}\right), 78.2(\mathrm{CH}), 111.8(\mathrm{C}), 158.9(\mathrm{C})$; MS (ESI) $\mathrm{m} / z$ (relative intensity) 236 $\left([\mathrm{M}+\mathrm{Na}]^{+}, 100\right), 214\left([\mathrm{MH}]^{+}, 2\right)$; HRMS (ESI) calcd for $\mathrm{C}_{10} \mathrm{H}_{15} \mathrm{O}_{4} \mathrm{~N}[\mathrm{M}+\mathrm{Na}]^{+}$236.0893, found 236.0892 .

Acetylated isoxazoline 18a. Following the general procedure for the acetylation of alcohol and purification with flash chromatography $\left(\mathrm{Et}_{2} \mathrm{O}\right)$, isoxazoline $18(34.3 \mathrm{mg}, 0.1608 \mathrm{mmol})$ was acetylated to afford isoxazoline 18a (41 mg, 99\%) as a white solid. The white solid was recrystallized from $\mathrm{Et}_{2} \mathrm{O}(\sim 4 \mathrm{~mL})$ with hexane $(\sim 10 \mathrm{~mL})$ as colorless crystals: $\mathrm{mp} 115-116^{\circ} \mathrm{C}$; $[\alpha]_{\mathrm{D}}^{20}$ $6.61^{\circ}$ ( $0.740, \mathrm{CHCl}_{3}$ ); $\mathrm{R}_{f} 0.13$ (hexane:Et $\left.2 \mathrm{O}, 1: 1\right) ;{ }^{1} \mathrm{H}$ NMR $\delta 1.39(3 \mathrm{H}, \mathrm{s}), 1.56(3 \mathrm{H}, \mathrm{s}), 1.65(1 \mathrm{H}$, $\operatorname{dtd}, J=14.4,5.1,0.9 \mathrm{~Hz}), 2.34(3 \mathrm{H}, 3), 2.43(1 \mathrm{H}, \mathrm{ddd}, J=13.5,11.4,9.3 \mathrm{~Hz}), 3.71(1 \mathrm{H}, \mathrm{tdd}, J=$ 11.7, 9.3, $3.0 \mathrm{~Hz}), 3.94(1 \mathrm{H}, \mathrm{dd}, J=12.8,8.4 \mathrm{~Hz}), 4.61(1 \mathrm{H}, \mathrm{ddd}, J=7.5,2.7,0.9 \mathrm{~Hz}), 4.68(1 \mathrm{H}$, dd, $J=10.8,8.1 \mathrm{~Hz}), 4.87(1 \mathrm{H}, \mathrm{ddd}, J=11.1,4.5,2.7 \mathrm{~Hz}), 5.06(1 \mathrm{H}, \mathrm{ddd}, J=11.1,4.5,2.7 \mathrm{~Hz}){ }^{13} \mathrm{C}$ 
NMR $\delta 21.7\left(\mathrm{CH}_{3}\right), 24.6\left(\mathrm{CH}_{3}\right), 26.5\left(\mathrm{CH}_{3}\right), 27.3\left(\mathrm{CH}_{2}\right), 40.5(\mathrm{CH}), 68.7(\mathrm{CH}), 69.8(\mathrm{CH}), 76.1$ $(\mathrm{CH}), 76.6\left(\mathrm{CH}_{2}\right), 112.0(\mathrm{C}), 156.7(\mathrm{C}), 170.7(\mathrm{C})$; MS (ESI) $\mathrm{m} / z$ (relative intensity) $278\left([\mathrm{M}+\mathrm{Na}]^{+}\right.$, 100), 256 ([MH] $\left.]^{+}, 19\right)$; HRMS (ESI) calcd for $\mathrm{C}_{11} \mathrm{H}_{17} \mathrm{O}_{5} \mathrm{~N}[\mathrm{M}+\mathrm{Na}]^{+} 278.0991$, found 278.1005.

Isoxazolines 21 and 22. Following the general procedure for oxime formation (method A), INOC reaction (method I) and purification with flash chromatography $\left(\mathrm{Et}_{2} \mathrm{O}\right)$, lactol $\mathbf{2 0}^{16}(251.3 \mathrm{mg}, 1.255$ mmol) was converted into isoxazoline 22 (128.5 $\mathrm{mg}$, 48\% overall yield from 20) as a white solid and isoxazoline 21 (127.9 $\mathrm{mg}, 48 \%$ overall yield from 20) as a colorless oil. Isoxazoline 21 was recrystallized from $\mathrm{Et}_{2} \mathrm{O}(\sim 3 \mathrm{~mL})$-hexane $(\sim 10 \mathrm{~mL})$ as colorless crystals: Data for 21 : $\mathrm{mp} 144-145{ }^{\circ} \mathrm{C}$; $[\alpha]_{\mathrm{D}}^{20}-34.1^{\circ}\left(c 0.62, \mathrm{CHCl}_{3}\right) ; \mathrm{R}_{f} 0.22\left(\mathrm{Et}_{2} \mathrm{O}\right)$; IR (thin film) 3340, 2987, 2885, 1634, 1360, 1249, $1060 \mathrm{~cm}^{-1}$; ${ }^{1} \mathrm{H}$ NMR $\delta 1.41(3 \mathrm{H}, \mathrm{s}), 1.52(3 \mathrm{H}, \mathrm{s}), 1.87(1 \mathrm{H}, \mathrm{ddd}, J=13.8,13.6,2.4 \mathrm{~Hz}), 1.9(1 \mathrm{H}$, br, s), $2.10(1 \mathrm{H}, \mathrm{ddd}, J=13.5,5.1,4.2 \mathrm{~Hz}), 3.60(1 \mathrm{H}, \mathrm{qd}, J=11.1,5.4 \mathrm{~Hz}), 3.93(1 \mathrm{H}, \mathrm{dd}, J=9.6,8.1$ $\mathrm{Hz}), 4.29(1 \mathrm{H}, \mathrm{ddd}, J=4.8,4.2,2.4 \mathrm{~Hz}), 4.39(1 \mathrm{H}, \mathrm{dd}, J=5.1,4.8 \mathrm{~Hz}), 4.56(1 \mathrm{H}, \mathrm{dd}, J=10.8,8.1$ $\mathrm{Hz}), 5.02(1 \mathrm{H}, \mathrm{d}, J=9.1 \mathrm{~Hz}) ;{ }^{13} \mathrm{C}$ NMR $\delta 26.9\left(\mathrm{CH}_{3}\right), 28.1\left(\mathrm{CH}_{3}\right), 30.2\left(\mathrm{CH}_{2}\right), 33.5(\mathrm{CH}), 68.0(\mathrm{CH})$, $71.4(\mathrm{CH}), 74.0\left(\mathrm{CH}_{2}\right), 79.0(\mathrm{CH}), 111.3(\mathrm{C}), 159.8(\mathrm{C})$; MS (ESI) $\mathrm{m} / z$ (relative intensity) 236 $\left([\mathrm{M}+\mathrm{Na}]^{+}, 100\right), 214\left([\mathrm{MH}]^{+}, 9\right)$; HRMS (ESI) calcd for $\mathrm{C}_{10} \mathrm{H}_{15} \mathrm{O}_{4} \mathrm{~N}[\mathrm{M}+\mathrm{Na}]^{+}$236.0893, found 236.0892 .

Data for 22: $[\alpha]_{\mathrm{D}}^{20} 73.8^{\circ}\left(c 0.94, \mathrm{CHCl}_{3}\right) ; \mathrm{R}_{f} 0.32\left(\mathrm{Et}_{2} \mathrm{O}\right)$; IR (thin film) 3408, 2984, 2935, 22340, 1635, 1454, 1435, 1375, 1240, 1219, 1157, 1062, $1001 \mathrm{~cm}^{-1} ;{ }^{1} \mathrm{H}$ NMR $\delta 1.39$ (3H, s), 1.43 (1H, ddd, $J=12.9,11.1,10.5 \mathrm{~Hz}), 1.52(3 \mathrm{H}, \mathrm{s}), 2.17(1 \mathrm{H}, \mathrm{ddd}, J=12.9,6.0,3.9 \mathrm{~Hz}), 2.84(1 \mathrm{H}, \mathrm{br}, \mathrm{s}), 3.50$ $(1 \mathrm{H}, \mathrm{qd}, J=10.5,6.0 \mathrm{~Hz}), 3.84(1 \mathrm{H}, \mathrm{ddd}, J=11.1,6.9,3.6 \mathrm{~Hz}), 4.00(1 \mathrm{H}, \mathrm{dd}, J=10.5,8.1), 4.10$ $(1 \mathrm{H}, \mathrm{dd}, J=6.9,6.0 \mathrm{~Hz}) 4.60(1 \mathrm{H}, \mathrm{dd}, J=10.5,8.1 \mathrm{~Hz}), 5.00(1 \mathrm{H}, \mathrm{d}, J=5.7 \mathrm{~Hz}) ;{ }^{13} \mathrm{C} \mathrm{NMR}\left(\mathrm{C}_{6} \mathrm{D}_{6}\right)$ $26.0\left(\mathrm{CH}_{3}\right), 28.1\left(\mathrm{CH}_{3}\right), 33.6\left(\mathrm{CH}_{2}\right), 42.6(\mathrm{CH}), 69.9(\mathrm{CH}), 70.9(\mathrm{CH}), 75.1\left(\mathrm{CH}_{2}\right), 82.4(\mathrm{CH})$, 111.2(C), $156.6(\mathrm{C})$; MS (ESI) $m / z$ (relative intensity) $236\left([\mathrm{M}+\mathrm{Na}]^{+}, 100\right), 214\left([\mathrm{MH}]^{+}, 33\right)$; HRMS (ESI) calcd for $\mathrm{C}_{10} \mathrm{H}_{15} \mathrm{O}_{4} \mathrm{~N}[\mathrm{M}+\mathrm{Na}]^{+} 236.0893$, found 236.0890.

\section{1,2,3-Trideoxy-5,6-O-[(2R,3R)-2,3-dimethoxybutan-2,3-dioxy]-L-gluco-oct-1-enitol 23 and} 1,2,3-trideoxy-5,6-O-[(2R,3R)-2,3-dimethoxybutan-2,3-dioxy]-L-manno-oct-1-enitol 37. To a solution of glycoside 36 (23.2 g, $65.5 \mathrm{mmol})$ in EtOH $(200 \mathrm{~mL})$ was added 10\% Pd-on-charcoal (696 mg, $0.655 \mathrm{mmol}$ ) and the mixture was stirred under an atmosphere of $\mathrm{H}_{2}$ (balloon). After stirring at room temperature under $\mathrm{H}_{2}$ for $12 \mathrm{~h}$, the mixture was filtered and the filtrate was concentrated to give lactol as a white solid. A solution of allylmagnesium bromide was generated with magnesium powder $(39.8 \mathrm{~g}, 1.64 \mathrm{~mol})$ and allyl bromide $(85.0 \mathrm{~mL}, 982 \mathrm{mmol})$ in $\mathrm{Et}_{2} \mathrm{O}(500$ $\mathrm{mL}$ ). The $\mathrm{Et}_{2} \mathrm{O}$ solution of allylmagnesium bromide was added dropwise to a stirred solution of the 
lactol in THF $(400 \mathrm{~mL})$ at $-78^{\circ} \mathrm{C}$ under $\mathrm{N}_{2}$ over $2 \mathrm{~h}$. After stirring at $-78^{\circ} \mathrm{C}$ for a further $1 \mathrm{~h}$ and then room temperature for $12 \mathrm{~h}$, the mixture was quenched with saturated $\mathrm{NH}_{4} \mathrm{Cl}$ solution and the aqueous phase was extracted with EtOAc $(2 \times 500 \mathrm{~mL})$. The combined organic extracts were dried over anhydrous $\mathrm{MgSO}_{4}$, and filtered. The filtrate was concentrated under reduced pressure and the residue was purified by flash chromatography $\left(\mathrm{CHCl}_{3}\right.$ :EtOAc, 1:4) to afford firstly alkene 37 (9.89 g, 49\% overall yield from 36) and secondly alkene 23 (9.77 g, 49\% overall yield from 36) as colorless oils: Data for 37: $[\alpha]_{\mathrm{D}}^{20}-147.5\left(c\right.$ 2.52, $\left.\mathrm{CHCl}_{3}\right)\left\{\right.$ lit. ${ }^{16}$ enantiomer of 37 had $[\alpha]_{\mathrm{D}}^{20}+148.7(c$ 1.43, $\left.\left.\mathrm{CHCl}_{3}\right)\right\} ; \mathrm{R}_{f} 0.33\left(\mathrm{CHCl}_{3}\right.$ :EtOAc, 1:3); IR (thin film) 3371, 2949, 1376, 1128, $1037 \mathrm{~cm}^{-1} ;{ }^{1} \mathrm{H}$ NMR $\delta 1.265-1.273(6 \mathrm{H}, 2 \mathrm{~s}), 2.15(1 \mathrm{H}, \mathrm{dt}, J=14.1,8.4 \mathrm{~Hz}), 2.81(1 \mathrm{H}, \mathrm{dddd}, J=14.1,6,2.7,1.5$ Hz), 3.01-3.34 (9H, m), $3.55(1 \mathrm{H}, \mathrm{ddd}, J=10.8,8.1,2.7 \mathrm{~Hz}), 3.70-3.90(5 \mathrm{H}, \mathrm{m}), 5.17-5.22(2 \mathrm{H}$, m), $5.84(1 \mathrm{H}$, dddd, $J=17.7,9.3,8.4,6 \mathrm{~Hz}) ;{ }^{1} \mathrm{H}$ NMR $\left(\mathrm{CDCl}_{3}-\mathrm{D}_{2} \mathrm{O}\right) \delta 1.26-1.27(6 \mathrm{H}, 2 \mathrm{~s}), 2.15(1 \mathrm{H}$, $\mathrm{dt}, J=14.4,9 \mathrm{~Hz}), 2.80(1 \mathrm{H}, \mathrm{dddd}, J=14.1,6,2.7,1.5 \mathrm{~Hz}), 3.25-3.26(6 \mathrm{H}, 2 \mathrm{~s}), 3.54(1 \mathrm{H}, \mathrm{dd}, J=$ 9.3, 8.1 Hz), 3.70-3.90 (5H, m), 5.16-5.22 (2H, m), $5.83(1 \mathrm{H}, \mathrm{dddd}, J=17.7,9.3,8.4,6 \mathrm{~Hz}) ;{ }^{13} \mathrm{C}$ NMR $\delta 17.7,17.8,37.8,48.6,63.8,70.6,70.9,73.6,73.9,99.8,99.0,119.3,135.0$; MS (ESI) $\mathrm{m} / z$ (relative intensity) $329\left([\mathrm{M}+\mathrm{Na}]^{+}, 100\right)$; HRMS (ESI) calcd for $\mathrm{C}_{14} \mathrm{H}_{26} \mathrm{O}_{7}[\mathrm{M}+\mathrm{Na}]^{+} 329.1571$, found 329.1575 .

Data for 23: $\left.[\alpha]_{\mathrm{D}}^{20}-148.9(c) 1.26, \mathrm{CHCl}_{3}\right)$ \{lit. ${ }^{16}$ enantiomer of 23 had $[\alpha]_{\mathrm{D}}^{20}+148.0$ (c 1.70, $\left.\left.\mathrm{CHCl}_{3}\right)\right\} ; \mathrm{R}_{f} 0.24\left(\mathrm{CHCl}_{3}\right.$ :EtOAc, 1:3); IR (thin film) 3393, 2949, 1377, 1127, $1037 \mathrm{~cm}^{-1} ;{ }^{1} \mathrm{H}$ NMR $\delta$ $1.25-1.28(6 \mathrm{H}, 2 \mathrm{~s}), 2.32-2.51(2 \mathrm{H}, 1 \mathrm{ddd}, J=14.4,6.9,5.7 \mathrm{~Hz}, 1 \mathrm{ddd}, J=14.4,8.4,7.2 \mathrm{~Hz}), 3.13$ $(3 \mathrm{H}, \mathrm{s}), 3.22-3.24(6 \mathrm{H}, 2 \mathrm{~s}), 3.68(1 \mathrm{H}, \mathrm{dd}, J=9.9,1.8 \mathrm{~Hz}), 3.72-3.81(3 \mathrm{H}, \mathrm{m}), 3.92(1 \mathrm{H}, \mathrm{ddd}, J=$ 8.1, 5.1, $1.8 \mathrm{~Hz}), 3.99$ (1H, dd, $J=9.9,6.3 \mathrm{~Hz}), 5.08-5.15(2 \mathrm{H}, \mathrm{m}), 5.83$ (1H, ddt, $J=17.1,10.2,6.9$ $\mathrm{Hz} ;{ }^{1} \mathrm{H}$ NMR $\left(\mathrm{CDCl}_{3}-\mathrm{D}_{2} \mathrm{O}\right) \delta 1.25-1.27$ (6H, 2s), 2.30-2.51 (2H, 1ddd, $J=14.1,6.6,5.4 \mathrm{~Hz}, 1 \mathrm{ddd}, J$ $=14.4,8.4,7.2 \mathrm{~Hz}), 3.22-3.24(6 \mathrm{H}, 2 \mathrm{~s}), 3.67(1 \mathrm{H}, \mathrm{dd}, J=9.9,1.8 \mathrm{~Hz}), 3.70-3.79(3 \mathrm{H}, \mathrm{m}), 3.91-$ $3.99(2 \mathrm{H}, 1 \mathrm{ddd}, J=8.1,5.1,1.8 \mathrm{~Hz}, 1 \mathrm{dd}, J=9.9,6.3 \mathrm{~Hz}), 5.06-5.15(2 \mathrm{H}, \mathrm{m}), 5.83(1 \mathrm{H}, \mathrm{ddt}, J=$ 17.1, 10.2, $6.9 \mathrm{~Hz}) ;{ }^{13} \mathrm{C}$ NMR $\delta 17.8,17.9,37.9,48.8,48.5,63.7,69.7,70.4,71.5,72.2,99.0,99.2$, 117.9, 135.5; MS (ESI) $\mathrm{m} / \mathrm{z}$ (relative intensity) 329 ([M+Na $\left.]^{+}, 100\right)$; HRMS (ESI) calcd for $\mathrm{C}_{14} \mathrm{H}_{26} \mathrm{O}_{7}[\mathrm{M}+\mathrm{Na}]^{+}$329.1571, found 329.1579.

Isoxazoline 24. Following the general procedure for glycol cleavage, formation of oxime (method A), INOC reaction (method I) and purification with flash chromatography (hexane:EtOAc, 2:1 to 1:2), alkene $23(220 \mathrm{mg}, 0.717 \mathrm{mmol})$ was converted into isoxazoline $24(172 \mathrm{mg}, 83 \%$ overall yield from 23) as a white solid: $\mathrm{mp} 233-234{ }^{\circ} \mathrm{C}$; $[\alpha]_{\mathrm{D}}^{20}-60.1\left(c\right.$ 2.34, $\left.\mathrm{CHCl}_{3}\right) ; \mathrm{R}_{f} 0.26$ (hexane:EtOAc, 1:2); IR (thin film) 3522, 2952, 1457, 1379, 1210, 1128, 1027, 870, 847, $650 \mathrm{~cm}^{-1}$; ${ }^{1} \mathrm{H}$ NMR $\delta 1.35(3 \mathrm{H}, \mathrm{s}), 1.39(3 \mathrm{H}, \mathrm{s}), 1.48(1 \mathrm{H}, \mathrm{q}, J=12 \mathrm{~Hz}), 2.35(1 \mathrm{H}, \mathrm{ddd}, J=12.3,6.3,4.5 \mathrm{~Hz})$, $2.56(1 \mathrm{H}, \mathrm{s}), 3.26(3 \mathrm{H}, \mathrm{s}), 3.30-3.43(4 \mathrm{H}, \mathrm{m}), 3.64(1 \mathrm{H}, \mathrm{t}, J=9.9 \mathrm{~Hz}), 3.88(2 \mathrm{H}, 1 \mathrm{dd}, J=10.5,8.4$ 
$\mathrm{Hz}, 1 \mathrm{~m}), 4.49(1 \mathrm{H}, \mathrm{d}, J=10.2 \mathrm{~Hz}), 4.57(1 \mathrm{H}, \mathrm{dd}, J=10.8,8.1 \mathrm{~Hz}) ;{ }^{13} \mathrm{C} \mathrm{NMR} \delta 17.9\left(\mathrm{CH}_{3}\right), 18.0$ $\left(\mathrm{CH}_{3}\right), 35.7\left(\mathrm{CH}_{2}\right), 45.6(\mathrm{CH}), 48.3\left(\mathrm{CH}_{3}\right), 48.8\left(\mathrm{CH}_{3}\right), 66.3(\mathrm{CH}), 68.8(\mathrm{CH}), 74.0(\mathrm{CH}), 75.5\left(\mathrm{CH}_{2}\right)$, 100.3 (C), 100.6 (C), 156.1 (C); MS (ESI) $m / z$ (relative intensity) 310 ([M+Na] $\left.]^{+}, 100\right)$; HRMS (ESI) calcd for $\mathrm{C}_{13} \mathrm{H}_{21} \mathrm{O}_{6} \mathrm{~N}[\mathrm{M}+\mathrm{Na}]^{+}$310.1261, found 310.1264; Anal. Calcd for $\mathrm{C}_{13} \mathrm{H}_{21} \mathrm{O}_{6} \mathrm{~N}$ : C, 54.35; $\mathrm{H}$, $7.37 ; \mathrm{N}, 4.87$, found: $\mathrm{C}, 53.90 ; \mathrm{H}, 7.27 ; \mathrm{N}, 4.63$.

Benzoates 25 and 27. Following the general procedure for glycol cleavage, diol $43^{17}$ (731 $\mathrm{mg}$, $2.27 \mathrm{mmol}$ ) was converted into crude aldehyde $(692 \mathrm{mg})$. It was then put to the next step without further purification. The crude aldehyde was dissolved in $30 \mathrm{~mL}$ THF, then a $0.1 \mathrm{~m} \mathrm{THF}$ solution of vinylmagnesium bromide $(21.0 \mathrm{~mL}, 2.1 \mathrm{mmol})$ was added dropwise at $-78^{\circ} \mathrm{C}$ under $\mathrm{N}_{2}$ over 30 min. After the addition, the reaction was quenched with saturated $\mathrm{NH}_{4} \mathrm{Cl}$ solution at $-78^{\circ} \mathrm{C}$. The aqueous phase was extracted with EtOAc $(3 \times 100 \mathrm{~mL})$. The combined organic extracts were dried over anhydrous $\mathrm{MgSO}_{4}$ and filtered. The filtrate was concentrated under reduced pressure and the residue was purified by flash chromatography (hexane: $\mathrm{Et}_{2} \mathrm{O}, 1: 1$ ) to afford firstly benzoate 25 (135 $\mathrm{mg}, 19 \%)$ and secondly benzoate 27 (157 mg, 21\%) as white solids. Data for 25: $\mathrm{mp} 139-141{ }^{\circ} \mathrm{C}$; $[\alpha]_{\mathrm{D}}^{20}+26.1\left(c 0.73, \mathrm{CHCl}_{3}\right.$ ); $\mathrm{R}_{f} 0.33$ (hexane: $\mathrm{Et}_{2} \mathrm{O}, 1: 1$ ); IR (thin film) 3504, 2988, 2928, 1724, 1265..1090, 949, $713 \mathrm{~cm}^{-1}$; ${ }^{1} \mathrm{H}$ NMR $\delta 1.34(3 \mathrm{H}, \mathrm{s}), 1.53(3 \mathrm{H}, \mathrm{s}), 2.63(1 \mathrm{H}, \mathrm{brs}), 4.02(1 \mathrm{H}, \mathrm{dd}, J=$ 7.5, $3.3 \mathrm{~Hz}), 4.95(1 \mathrm{H}, \mathrm{t}, J=6 \mathrm{~Hz}), 4.83(1 \mathrm{H}, \mathrm{dd}, J=6.0,3.3 \mathrm{~Hz}), 4.88(1 \mathrm{H}, \mathrm{d}, J=6 \mathrm{~Hz}), 5.29(1 \mathrm{H}$, $\mathrm{dt}, J=10.5,1.5 \mathrm{~Hz}), 5.53(1 \mathrm{H}, \mathrm{dt}, J=17.4,1.5 \mathrm{~Hz}), 6.01(1 \mathrm{H}, \mathrm{ddd}, J=17.4,10.8,5.1 \mathrm{~Hz}), 6.44$ $(1 \mathrm{H}, \mathrm{s}), 7.41-7.47(2 \mathrm{H}, \mathrm{m}), 7.55-7.61(1 \mathrm{H}, \mathrm{m}), 7.98-8.01(2 \mathrm{H}, \mathrm{m}) ;{ }^{13} \mathrm{C} \mathrm{NMR} \delta 25.2\left(\mathrm{CH}_{3}\right), 26.5$ $\left(\mathrm{CH}_{3}\right), 71.3(\mathrm{CH}), 80.0(\mathrm{CH}), 85.9(\mathrm{CH}), 86.1(\mathrm{CH}), 101.7(\mathrm{CH}), 113.9(\mathrm{C}), 117.7\left(\mathrm{CH}_{2}\right), 128.9$ (CH), $129.9(\mathrm{C}), 130.3(\mathrm{CH}), 133.9(\mathrm{CH}), 135.5(\mathrm{CH}), 165.5(\mathrm{C})$; MS (ESI) $\mathrm{m} / z$ (relative intensity) $343\left([\mathrm{M}+\mathrm{Na}]^{+}, 100\right), 216$ (1), 141 (1), 123 (1); HRMS (ESI) calcd for $\mathrm{C}_{17} \mathrm{H}_{20} \mathrm{O}_{6}[\mathrm{M}+\mathrm{Na}]^{+} 343.1152$, found 343.1156 .

Data for 27: $\mathrm{mp} 99-102{ }^{\circ} \mathrm{C}$; $[\alpha]_{\mathrm{D}}^{20}+44.6\left(c 1.08, \mathrm{CHCl}_{3}\right)$; $\mathrm{R}_{f} 0.23$ (hexane:Et $\left.\mathrm{E}_{2} \mathrm{O}, 1: 1\right)$; IR (thin film) 3515, 2988, 2939, 1727, 1263, 1092, 949, $713 \mathrm{~cm}^{-1}$; ${ }^{1} \mathrm{H}$ NMR $\delta 1.37$ (3H, s), $1.56(3 \mathrm{H}, \mathrm{s}), 2.73(1 \mathrm{H}$, $\mathrm{d}, J=6 \mathrm{~Hz}), 4.08(1 \mathrm{H}, \mathrm{dd}, J=7.5,3.6 \mathrm{~Hz}), 4.50(1 \mathrm{H}, \mathrm{q}, J=6 \mathrm{~Hz}), 4.86(1 \mathrm{H}, \mathrm{d}, J=6 \mathrm{~Hz}), 5.00(1 \mathrm{H}$, $\mathrm{dd}, J=6,3.9 \mathrm{~Hz}), 5.26(1 \mathrm{H}, \mathrm{dt}, J=11.7,1.2 \mathrm{~Hz}), 5.44(1 \mathrm{H}, \mathrm{dt}, J=17.4,1.5 \mathrm{~Hz}), 6.04(1 \mathrm{H}, \mathrm{ddd}, J=$ 17.1, 10.5, $5.4 \mathrm{~Hz}), 6.45(1 \mathrm{H}, \mathrm{s}), 7.41-7.46(2 \mathrm{H}, \mathrm{m}), 7.56-7.60(1 \mathrm{H}, \mathrm{m}), 7.98-8.01(2 \mathrm{H}, \mathrm{m}) ;{ }^{13} \mathrm{C}$ NMR $\delta 25.1\left(\mathrm{CH}_{3}\right), 26.4\left(\mathrm{CH}_{3}\right), 71.1(\mathrm{CH}), 80.2(\mathrm{CH}), 84.2(\mathrm{CH}), 85.3(\mathrm{CH}), 101.7(\mathrm{CH}), 113.8(\mathrm{C})$, $116.9\left(\mathrm{CH}_{2}\right), 128.9(\mathrm{CH}), 129.9(\mathrm{C}), 130.2(\mathrm{CH}), 133.9(\mathrm{CH}), 137.7(\mathrm{CH}), 165.3(\mathrm{C})$; MS (ESI) $m / z$ (relative intensity) $343\left([\mathrm{M}+\mathrm{Na}]^{+}, 100\right), 216$ (3), 141 (5), 123 (2); HRMS (ESI) calcd for $\mathrm{C}_{17} \mathrm{H}_{20} \mathrm{O}_{6}$ $[\mathrm{M}+\mathrm{Na}]^{+}$343.1152, found 343.1161. 
Isoxazoline 26. To a solution of the benzoate $25(39.0 \mathrm{mg}, 0.122 \mathrm{mmol})$ in $\mathrm{MeOH}(4 \mathrm{~mL})$ was added solid $\mathrm{K}_{2} \mathrm{CO}_{3}(3.0 \mathrm{mg}, 0.024 \mathrm{mmol})$ at room temperature and then stirred for $2 \mathrm{~h}$. The solvent was evaporated and the inorganic salt was removed by gel filtration. Concentration of the filtrate afforded a lactol which was used in the next step without further purification. Following the general procedure for the formation of oxime (method A), INOC reaction (method I) and purification with flash chromatography (hexane: $\left.\mathrm{Et}_{2} \mathrm{O}, 1: 3\right)$, the lactol was converted into isoxazoline 26 (22.0 $\mathrm{mg}$, $77 \%$ overall yield from 25$)$ as a white solid: $\mathrm{mp} 169-171{ }^{\circ} \mathrm{C} ;[\alpha]_{\mathrm{D}}^{20}-5.64\left(c 0.52, \mathrm{CHCl}_{3}\right) ; \mathrm{R}_{f} 0.33$ (EtOAc); IR (thin film) 3374, 2918, 1624, 1462, 1383, 1219, 1053, $863 \mathrm{~cm}^{-1} ;{ }^{1} \mathrm{H}$ NMR $\delta 1.43(3 \mathrm{H}$, s), $1.57(3 \mathrm{H}, \mathrm{s}), 3.18(1 \mathrm{H}, \mathrm{brs}), 3.28(1 \mathrm{H}, \mathrm{brs}), 3.37(1 \mathrm{H}, \mathrm{t}, J=9.9 \mathrm{~Hz}), 3.53(1 \mathrm{H}, \mathrm{dt}, J=10.5,8.1$ $\mathrm{Hz}), 3.66(1 \mathrm{H}, \mathrm{t}, J=8.4 \mathrm{~Hz}), 4.05(1 \mathrm{H}, \mathrm{dd}, J=7.8,5.4 \mathrm{~Hz}), 4.36(1 \mathrm{H}, \mathrm{t}, J=8.4 \mathrm{~Hz}), 4.63(1 \mathrm{H}, \mathrm{dd}, J$ $=10.5,8.7 \mathrm{~Hz}), 4.98(1 \mathrm{H}, \mathrm{d}, J=5.4 \mathrm{~Hz}) ;{ }^{13} \mathrm{C} \mathrm{NMR} \delta 26.7\left(\mathrm{CH}_{3}\right), 28.6\left(\mathrm{CH}_{3}\right), 50.8(\mathrm{CH}), 70.0(\mathrm{CH})$, $73.6(\mathrm{CH}), 73.7\left(\mathrm{CH}_{2}\right), 77.3(\mathrm{CH}), 80.9(\mathrm{CH}), 112.4(\mathrm{C}), 153.4(\mathrm{C})$; MS (ESI) $m / z$ (relative intensity) $252\left([\mathrm{MH}]^{+}, 100\right), 230$ (62); HRMS (ESI) calcd for $\mathrm{C}_{10} \mathrm{H}_{15} \mathrm{~N}_{1} \mathrm{O}_{5}[\mathrm{MH}]^{+}$252.0842, found 252.0843.

Acetylated isoxazolines 28 and 29. Following the same procedure for the formation of isoxazoline 26, benzoate $27(46.0 \mathrm{mg}, 0.143 \mathrm{mmol})$ was converted into a mixture of isoxazolines as colorless oils. Following the general procedure for the acetylation of alcohol and purification with flash chromatography (hexane: $\mathrm{Et}_{2} \mathrm{O}, 1: 1$ to $1: 3$ ), the isoxazolines were acetylated to afford acetate $28(10.6 \mathrm{mg}, 24 \%)$ and acetate $29(21.8 \mathrm{mg}, 49 \%)$ as colorless oils: Data for 28: $[\alpha]_{\mathrm{D}}^{20}+14.9(c 0.91$, $\mathrm{CHCl}_{3}$ ); $\mathrm{R}_{f} 0.47$ (hexane:Et ${ }_{2} \mathrm{O}, 1: 3$ ); IR (thin film) 2988, 2923, 1750, 1635, 1371, 1238, 1219, 1068 $\mathrm{cm}^{-1} ;{ }^{1} \mathrm{H}$ NMR $\delta 1.43(3 \mathrm{H}, \mathrm{s}), 1.56(3 \mathrm{H}, \mathrm{s}), 2.08(3 \mathrm{H}, \mathrm{s}), 2.10(3 \mathrm{H}, \mathrm{s}), 3.88(1 \mathrm{H}, \mathrm{ddd}, J=11.7,8.7,3$ $\mathrm{Hz}), 4.06(1 \mathrm{H}, \mathrm{t}, J=8.7 \mathrm{~Hz}), 4.39(1 \mathrm{H}, \mathrm{dd}, J=8.1,5.4 \mathrm{~Hz}), 4.52(1 \mathrm{H}, \mathrm{dd}, J=11.7,8.7 \mathrm{~Hz}), 5.02$ $(1 \mathrm{H}, \mathrm{dd}, J=8.1,2.4 \mathrm{~Hz}), 5.12(1 \mathrm{H}, \mathrm{d}, J=5.4 \mathrm{~Hz}), 5.44(1 \mathrm{H}, \mathrm{t}, J=2.7 \mathrm{~Hz}) ;{ }^{13} \mathrm{C} \mathrm{NMR} \delta 21.1\left(\mathrm{CH}_{3}\right)$, $21.3\left(\mathrm{CH}_{3}\right), 26.7\left(\mathrm{CH}_{3}\right), 28.3\left(\mathrm{CH}_{3}\right), 47.7(\mathrm{CH}), 69.2(\mathrm{CH}), 69.7(\mathrm{CH}), 70.5\left(\mathrm{CH}_{2}\right), 73.8(\mathrm{CH}), 77.2$ $(\mathrm{CH}), 112.5(\mathrm{C}), 152.5(\mathrm{C}), 170.6(\mathrm{C}), 170.9(\mathrm{C})$; MS (ESI) $\mathrm{m} / z$ (relative intensity) $336\left([\mathrm{M}+\mathrm{Na}]^{+}\right.$, 100), 301 (7), 256 (7), 231 (4); HRMS (ESI) calcd for $\mathrm{C}_{14} \mathrm{H}_{19} \mathrm{O}_{7} \mathrm{~N}_{1}[\mathrm{M}+\mathrm{Na}]^{+}$336.1054, found 336.1053 .

Data for 29: $[\alpha]_{\mathrm{D}}^{20}-6.9\left(c 1.09, \mathrm{CHCl}_{3}\right.$ ); $\mathrm{R}_{f} 0.27$ (hexane: $\mathrm{Et}_{2} \mathrm{O}, 1: 3$ ); IR (thin film) 2988, 2937, 1752, 1434, 1373, 1223, 1054, $851 \mathrm{~cm}^{-1} ;{ }^{1} \mathrm{H}$ NMR $\delta 1.39(3 \mathrm{H}, \mathrm{s}), 1.52(3 \mathrm{H}, \mathrm{s}), 2.03(3 \mathrm{H}, \mathrm{s}), 2.14(3 \mathrm{H}, \mathrm{s})$, $3.74(1 \mathrm{H}, \mathrm{q}, J=10.8 \mathrm{~Hz}), 4.10(1 \mathrm{H}, \mathrm{t}, J=8.7 \mathrm{~Hz}), 4.28(1 \mathrm{H}, \mathrm{dd}, J=5.1,3.6 \mathrm{~Hz}), 4.54(1 \mathrm{H}, \mathrm{dd}, J=$ 10.8, 8.7 Hz), $4.99(1 \mathrm{H}, \mathrm{dd}, J=4.8,0.9 \mathrm{~Hz}), 5.14(1 \mathrm{H}, \mathrm{dd}, J=10.8,2.7 \mathrm{~Hz}), 5.72(1 \mathrm{H}, \mathrm{t}, J=3.3$ $\mathrm{Hz}) ;{ }^{13} \mathrm{C}$ NMR $\delta 21.2\left(\mathrm{CH}_{3}\right), 26.6\left(\mathrm{CH}_{3}\right), 27.9\left(\mathrm{CH}_{3}\right), 48.6(\mathrm{CH}), 69.2(\mathrm{CH}), 70.8(\mathrm{CH}), 71.6(\mathrm{CH})$, $72.7\left(\mathrm{CH}_{2}\right), 76.4(\mathrm{CH}), 112.2(\mathrm{C}), 156.2(\mathrm{C}), 169.7$ (C), 170.1 (C); MS (ESI) $m / z$ (relative intensity) $336\left([\mathrm{M}+\mathrm{Na}]^{+}, 100\right), 314(7), 256(8)$; HRMS (ESI) calcd for $\mathrm{C}_{14} \mathrm{H}_{19} \mathrm{O}_{7} \mathrm{~N}_{1}[\mathrm{M}+\mathrm{Na}]^{+} 336.1054$, found 336.1059 . 
Lactol 30. To a solution of alkene $\mathbf{4 4}^{18}(359 \mathrm{mg}, 1.40 \mathrm{mmol})$ in $\mathrm{CH}_{2} \mathrm{Cl}_{2}(3 \mathrm{~mL})$ was added TFA (2 $\mathrm{mL})$ and $\mathrm{H}_{2} \mathrm{O}(0.5 \mathrm{~mL})$, and the mixture was stirred at room temperature for $48 \mathrm{~h}$. The solvent was removed under reduced pressure and flash chromatography $\left(\mathrm{CHCl}_{3}: \mathrm{MeOH}, 10: 1\right)$ of the residue afforded lactol $30(227 \mathrm{mg}, 92 \%)$ as a colorless oil: $[\alpha]_{\mathrm{D}}^{20}+57.2$ (c 2.2, $\left.\mathrm{MeOH}\right) ; \mathrm{R}_{f} 0.23$ $\left(\mathrm{CHCl}_{3}: \mathrm{MeOH}: 30 \%\right.$ aq. $\left.\mathrm{NH}_{3}, 2: 1: 0.2\right) ;{ }^{1} \mathrm{H}$ NMR $\left(\mathrm{CD}_{3} \mathrm{OD}\right) \delta 3.19-3.25(0.7 \mathrm{H}, \mathrm{m}), 3.36-3.43(2 \mathrm{H}$, m), 3.64-3.72 (3.6H, m), $3.92(1.2 \mathrm{H}, \mathrm{d}, J=5.7 \mathrm{~Hz}), 4.36(1 \mathrm{H}, \mathrm{d}, J=7.2 \mathrm{~Hz}), 4.40(0.9 \mathrm{H}, \mathrm{d}, J=5.1$ $\mathrm{Hz}), 5.06-5.11(3.5 \mathrm{H}, \mathrm{m}), 5.18-5.28(2.4 \mathrm{H}, \mathrm{m}), 5.79-5.92(2.3 \mathrm{H}, \mathrm{m}) ;{ }^{13} \mathrm{C} \mathrm{NMR}\left(\mathrm{CD}_{3} \mathrm{OD}\right) \delta 70.1$ $(\mathrm{CH}), 71.2(\mathrm{CH}), 72.2(\mathrm{CH}), 72.9(\mathrm{CH}), 73.5(\mathrm{CH}), 74.9(\mathrm{CH}), 77.0(\mathrm{CH}), 94.1(\mathrm{CH}), 98.5(\mathrm{CH})$, $116.8\left(\mathrm{CH}_{2}\right), 117.1\left(\mathrm{CH}_{2}\right), 136.1(\mathrm{CH}), 136.7(\mathrm{CH})$; MS (ESI) $m / z$ (relative intensity) $199\left([\mathrm{M}+\mathrm{Na}]^{+}\right.$, 100); HRMS (ESI) calcd for $\mathrm{C}_{7} \mathrm{H}_{12} \mathrm{O}_{5}[\mathrm{M}+\mathrm{Na}]^{+}$199.0577, found 199.0583.

Isoxazoline 31. According to the general procedure for the formation of oxime (method B), INOC reaction (method I) and purification with flash chromatography $\left(\mathrm{CHCl}_{3}: \mathrm{MeOH}, 10: 1\right)$, lactol 30 (41.8 mg, $0.237 \mathrm{mmol})$ was converted into isoxazoline $31(32.0 \mathrm{mg}, 72 \%)$ as a colorless oil: $[\alpha]_{\mathrm{D}}^{20}$ -35.3 (c 0.54, $\left.\mathrm{CHCl}_{3}\right) ; \mathrm{R}_{f} 0.4\left(\mathrm{CHCl}_{3}: \mathrm{MeOH}: 30 \%\right.$ aq. $\left.\mathrm{NH}_{3}, 1: 1: 0.2\right) ;{ }^{1} \mathrm{H} \mathrm{NMR}\left(\mathrm{CD}_{3} \mathrm{OD}\right) \delta 3.42(1 \mathrm{H}$, q, $J=9.75 \mathrm{~Hz}), 3.57(1 \mathrm{H}, \mathrm{t}, J=9.6 \mathrm{~Hz}), 3.71(1 \mathrm{H}, \mathrm{dd}, J=9.5,3 \mathrm{~Hz}), 3.91(1 \mathrm{H}, \mathrm{t}, J=3 \mathrm{~Hz}), 4.05$ $(1 \mathrm{H}, \mathrm{t}, J=8.4 \mathrm{~Hz}), 4.34(1 \mathrm{H}, \mathrm{d}, J=3.3 \mathrm{~Hz}), 4.43(1 \mathrm{H}, \mathrm{dd}, J=10.5,8.4 \mathrm{~Hz}) ;{ }^{13} \mathrm{C} \mathrm{NMR}\left(\mathrm{CD}_{3} \mathrm{OD}\right) \delta$ $52.2(\mathrm{CH}), 66.7(\mathrm{CH}), 72.1(\mathrm{CH}), 73.2\left(\mathrm{CH}_{2}\right), 73.7(\mathrm{CH}), 73.7(\mathrm{CH}), 158.3(\mathrm{C}) ; \mathrm{MS}(\mathrm{ESI}) \mathrm{m} / \mathrm{z}$ (relative intensity) $212\left([\mathrm{M}+\mathrm{Na}]^{+}, 100\right), 190$ (16), 110 (14); HRMS (ESI) calcd for $\mathrm{C}_{7} \mathrm{H}_{11} \mathrm{~N}_{1} \mathrm{O}_{5}$ $[\mathrm{M}+\mathrm{Na}]^{+}$212.0529, found 212.0533.

Alkene 33 and diastereomer 33a. A $1 \mathrm{M}$ THF solution of allylmagnesium bromide $(3.38 \mathrm{~mL}$, $3.38 \mathrm{mmol}$ ) was added dropwise to a stirred solution of acetonide $\mathbf{3 2}^{10}$ (110 $\left.\mathrm{mg}, 0.423 \mathrm{mmol}\right)$ in $\mathrm{Et}_{2} \mathrm{O}(20 \mathrm{~mL})$ at $-78^{\circ} \mathrm{C}$ under $\mathrm{N}_{2}$ over $30 \mathrm{~min}$. The reaction mixture was stirred for $1 \mathrm{~h}$ and then raised to $-30{ }^{\circ} \mathrm{C}$ and stirred a further $12 \mathrm{~h}$. The mixture was quenched with saturated $\mathrm{NH}_{4} \mathrm{Cl}$ solution and the aqueous phase was extracted with $\mathrm{Et}_{2} \mathrm{O}(2 \times 30 \mathrm{~mL})$. The combined organic extracts were dried over anhydrous $\mathrm{MgSO}_{4}$, and filtered. The filtrate was concentrated under reduced pressure and the residue was purified by flash chromatography (hexane: $\mathrm{Et}_{2} \mathrm{O}, 2: 1$ ) to afford firstly alkene 33 (107 $\mathrm{mg}, 83 \%)$ and secondly diastereomer 33a $(20.6 \mathrm{mg}, 16 \%)$ as colorless oils. Data for 33: $[\alpha]_{\mathrm{D}}^{20}-32.3$ (c 0.88, $\mathrm{CHCl}_{3}$ ); $\mathrm{R}_{f} 0.29$ (hexane:Et $2 \mathrm{O}, 1: 1$ ); IR (thin film) 3466, 2987, 1373, 1216, 1063, $849 \mathrm{~cm}^{-1}$; ${ }^{1} \mathrm{H}$ NMR $\delta 1.35(3 \mathrm{H}, \mathrm{s}), 1.37(3 \mathrm{H}, \mathrm{s}), 1.40(3 \mathrm{H}, \mathrm{s}), 1.48(3 \mathrm{H}, \mathrm{s}), 2.19(1 \mathrm{H}, \mathrm{dt}, J=15,8.1 \mathrm{~Hz}), 2.53-$ $2.61(2 \mathrm{H}, 1 \mathrm{~m} ; 1 \mathrm{~d}, J=3.6 \mathrm{~Hz}), 2.78(1 \mathrm{H}, \mathrm{d}, J=7.2 \mathrm{~Hz}), 3.90-4.13(6 \mathrm{H}, \mathrm{m}), 4.39(1 \mathrm{H}, \mathrm{dd}, J=6,1.2$ $\mathrm{Hz}), 5.16-5.21(2 \mathrm{H}, \mathrm{m}), 5.85(1 \mathrm{H}, \mathrm{dddd}, J=17.7,9.6,8.1,6.6 \mathrm{~Hz}) ;{ }^{1} \mathrm{H}$ NMR $\left(\mathrm{CDCl}_{3}-\mathrm{D}_{2} \mathrm{O}\right) \delta 1.35-$ 
$1.37(6 \mathrm{H}, 2 \mathrm{~s}), 1.40(3 \mathrm{H}, \mathrm{s}), 1.48(3 \mathrm{H}, \mathrm{s}), 2.19(1 \mathrm{H}, \mathrm{dt}, J=15,7.8 \mathrm{~Hz}), 2.54-2.61(1 \mathrm{H}, \mathrm{dd}, J=14.1$, $6.3 \mathrm{~Hz}), 3.90-3.93(1 \mathrm{H}, \mathrm{m}), 3.99-4.13(5 \mathrm{H}, \mathrm{m}), 4.38(1 \mathrm{H}, \mathrm{dd}, J=5.7,1.2 \mathrm{~Hz}), 5.16-5.21(2 \mathrm{H}, \mathrm{m})$, 5.86 (1H, dddd, $J=17.7,9.6,8.1,6.6 \mathrm{~Hz}) ;{ }^{13} \mathrm{C} \mathrm{NMR} \delta 25.3,25.8,27.2,27.3,39.3,67.5,68.9,70.0$, 76.2, 76.7, 79.4, 108.6, 109.7, 119.4, 134.3; MS (FAB) $\mathrm{m} / z$ (relative intensity) $303\left([\mathrm{MH}]^{+}, 100\right)$, 185 (100), 93 (98); HRMS (FAB) calcd for $\mathrm{C}_{15} \mathrm{H}_{26} \mathrm{O}_{6}[\mathrm{MH}]^{+}$303.1802, found 303.1809.

Data for diastereomer 33a: $[\alpha]_{\mathrm{D}}^{20}-20.2\left(c 0.96, \mathrm{CHCl}_{3}\right)$; $\mathrm{R}_{f} 0.21$ (hexane: $\left.\mathrm{Et}_{2} \mathrm{O}, 1: 1\right)$; IR (thin film) 3394, 2986, 2936, 1372, 1215, 1068, $849 \mathrm{~cm}^{-1}$; ${ }^{1} \mathrm{H}$ NMR $\delta 1.35$ (3H, s), 1.39-1.40 (6H, 2s), 1.54 (3H, s), 2.31-2.50 (2H, 1dddt, $J=14.1,7.2,6,0.9 \mathrm{~Hz} ; 1 \mathrm{dt}, J=14.1,6.9 \mathrm{~Hz}), 2.90(1 \mathrm{H}, \mathrm{br}$ s), 3.61 $(1 \mathrm{H}, \mathrm{d}, J=7.2 \mathrm{~Hz}), 3.88(1 \mathrm{H}$, br s), $4.00-4.13(3 \mathrm{H}, \mathrm{m}), 4.16(1 \mathrm{H}, \mathrm{dd}, J=7.5,1.5 \mathrm{~Hz}), 4.40(1 \mathrm{H}, \mathrm{d}, J$ $=7.5 \mathrm{~Hz}), 5.13-5.20(2 \mathrm{H}, \mathrm{m}), 5.86(1 \mathrm{H}, \mathrm{ddt}, J=17.4,10.2,7.2 \mathrm{~Hz}) ;{ }^{1} \mathrm{H}$ NMR $\left(\mathrm{CDCl}_{3}-\mathrm{D}_{2} \mathrm{O}\right) \delta 1.35$ $(3 \mathrm{H}, \mathrm{s}), 1.39-1.40(6 \mathrm{H}, 2 \mathrm{~s}), 1.54(3 \mathrm{H}, \mathrm{s}), 2.31-2.50(2 \mathrm{H}, 1 \mathrm{dddt}, J=14.1,7.2,6,1.2 \mathrm{~Hz} ; 1 \mathrm{dt}, J=$ $14.1,6.9 \mathrm{~Hz}), 3.60(1 \mathrm{H}, \mathrm{d}, J=7.5 \mathrm{~Hz}), 3.88(1 \mathrm{H}, \mathrm{ddd}, J=7.5,5.7,1.5 \mathrm{~Hz}), 4.00-4.13(3 \mathrm{H}, \mathrm{m}), 4.16$ $(1 \mathrm{H}, \mathrm{dd}, J=7.8,1.5 \mathrm{~Hz}), 4.40(1 \mathrm{H}, \mathrm{dd}, J=7.5,0.6 \mathrm{~Hz}), 5.13-5.21(2 \mathrm{H}, \mathrm{m}), 5.86(1 \mathrm{H}, \mathrm{ddt}, J=17.1$, 10.2, 7.2 Hz); ${ }^{13} \mathrm{C}$ NMR $\delta 25.0,25.7,26.6,27.3,40.2,67.8,68.9,70.8,76.2,76.3,78.5,108.8$, 109.7, 118.8, 134.5; MS (ESI) $\mathrm{m} / z$ (relative intensity) $325\left([\mathrm{M}+\mathrm{Na}]^{+}, 100\right)$; HRMS (ESI) calcd for $\mathrm{C}_{15} \mathrm{H}_{26} \mathrm{O}_{6}[\mathrm{M}+\mathrm{Na}]^{+}$325.1622, found 325.1623.

Lactol 34. Following the selective hydrolysis-glycol cleavage oxidation procedure for the formation of lactol 7 and purification with flash chromatography (hexane: $\mathrm{Et}_{2} \mathrm{O}, 3: 2$ ), alkene 33 (41.0 $\mathrm{mg}, 0.14 \mathrm{mmol})$ was converted to lactol $34(21.4 \mathrm{mg}, 79 \%)$ as a colorless oil: $[\alpha]_{\mathrm{D}}^{20}-5.3(c) 2.53$, $\mathrm{CHCl}_{3}$ ); $\mathrm{R}_{f} 0.45$ (hexane:Et ${ }_{2} \mathrm{O}, 2: 3$ ); IR (thin film) 3434, 3079, 2984, 2941, 1642, 1438, 1375, 1211 , $1075 \mathrm{~cm}^{-1}$; ${ }^{1} \mathrm{H}$ NMR (mixture of $\alpha$ and $\beta$ isomer with ratio $\left.\alpha: \beta=1: 5.6\right) \delta 1.31(3.0 \mathrm{H}, \mathrm{s}), 1.38(0.54 \mathrm{H}$, s), $1.47(3.0 \mathrm{H}, \mathrm{s}), 1.56(0.54 \mathrm{H}, \mathrm{s}), 2.21-2.53(2.36 \mathrm{H}, \mathrm{m}), 3.08(1.0 \mathrm{H}, \mathrm{d}, J=2.1 \mathrm{~Hz}), 3.92(0.18 \mathrm{H}, \mathrm{d}$, $J=9 \mathrm{~Hz}), 4.14(0.18 \mathrm{H}, \mathrm{td}, J=9,2.7 \mathrm{~Hz}), 4.25(1.0 \mathrm{H}, \mathrm{t}, J=8.1 \mathrm{~Hz}), 4.49(0.18 \mathrm{H}, \mathrm{dd}, J=6.6,2.7$ $\mathrm{Hz}), 4.61(1.0 \mathrm{H}, \mathrm{d}, J=6 \mathrm{~Hz}), 4.64(1.0 \mathrm{H}, \mathrm{d}, J=6 \mathrm{~Hz}), 5.10(1.18 \mathrm{H}, \mathrm{s}), 5.14(1.18 \mathrm{H}, \mathrm{d}, J=4.2 \mathrm{~Hz})$, $5.28(0.18 \mathrm{H}, \mathrm{dd}, J=9.3,4.2 \mathrm{~Hz}), 5.44(1.0 \mathrm{H}, \mathrm{d}, J=2.4 \mathrm{~Hz}), 5.72-5.87(1.18 \mathrm{H}, \mathrm{m}) ;{ }^{13} \mathrm{C} \mathrm{NMR} \delta$ 25.4, 26.7, 26.9, 37.5, 40.4, 79.9, 80.3, 83.5, 84.1, 86.5, 86.8, 96.3, 103.6, 112.8, 115.2, 118.2, 118.7, 133.6, 134.5; MS (ESI) $m / z$ (relative intensity) $223\left([\mathrm{M}+\mathrm{Na}]^{+}, 100\right), 185\left(\left[\mathrm{M}-\mathrm{CH}_{3}\right]^{+}, 7\right), 183([\mathrm{M}-$ $\left.\mathrm{OH}]^{+}, 3\right), 149$ (3); HRMS (ESI) calcd for $\mathrm{C}_{10} \mathrm{H}_{16} \mathrm{O}_{4}[\mathrm{M}+\mathrm{Na}]^{+}$223.0941, found 223.0945.

Benzyl-2,3-O-[(2R,3R)-2,3-dimethoxybutan-2,3-dioxy]- $\beta$-L-arabinopyranoside $36 . \quad$ To a suspension of benzyl- $\beta$-L-arabinopyranoside $35^{12}(111 \mathrm{mg}, 0.461 \mathrm{mmol})$ in methanol $(10 \mathrm{~mL})$ were added 2,2,3,3-tetramethoxybutane $(107 \mathrm{mg}, 0.600 \mathrm{mmol})$, trimethylorthoformate $(0.20 \mathrm{~mL}, 1.84$ mmol) and ( \pm )-10-camphorsulfonic acid $(10.7 \mathrm{mg}, 0.046 \mathrm{mmol})$ and the mixture was heated under 
reflux for $8 \mathrm{~h}$. Powered $\mathrm{NaHCO}_{3}(0.1 \mathrm{~g})$ was then added to the cooled reaction mixture which was stirred for $5 \mathrm{~h}$. The mixture was filtered and the filtrate was concentrated under reduced pressure. The residue was dissolved in EtOAc and the solution was washed with saturated $\mathrm{NaHCO}_{3}$ solution. The organic layer was dried over anhydrous $\mathrm{MgSO}_{4}$, and filtered. The filtrate was concentrated under reduced pressure and the crude residue was purified by flash chromatography (hexane:EtOAc, 1:1) to afford glycoside $36(125 \mathrm{mg}, 77 \%)$ as a white solid: $\mathrm{mp} 143-144{ }^{\circ} \mathrm{C}$ \{ lit. $^{16}$ enantiomer of 36 had mp $\left.144-145{ }^{\circ} \mathrm{C}\right\} ;[\alpha]_{\mathrm{D}}^{20}+4.10\left(c\right.$ 2.11, $\left.\mathrm{CHCl}_{3}\right)\left\{\right.$ lit. $^{16}$ enantiomer of 36 had $[\alpha]_{\mathrm{D}}^{20}-3.94$ (c 1.27, $\mathrm{CHCl}_{3}$ )\}; $\mathrm{R}_{f} 0.33$ (hexane:EtOAc, 1:1); IR (thin film) 3480, 2945, 1455, 1378, 1136, 1038, $885 \mathrm{~cm}^{-1}$; ${ }^{1} \mathrm{H}$ NMR $\delta$ 1.31-1.33 (6H, 2s), $2.02(1 \mathrm{H}, \mathrm{br} \mathrm{s}), 3.22(3 \mathrm{H}, \mathrm{s}), 3.26(3 \mathrm{H}, \mathrm{s}), 3.70(1 \mathrm{H}, \mathrm{dd}, J=12.9,1.8$ $\mathrm{Hz}), 3.82(1 \mathrm{H}, \mathrm{dd}, J=12.9,1.5 \mathrm{~Hz}), 3.92-3.93(1 \mathrm{H}, \mathrm{m}), 4.14(1 \mathrm{H}, \mathrm{dd}, J=10.5,3 \mathrm{~Hz}), 4.19(1 \mathrm{H}, \mathrm{dd}$, $J=10.5,3 \mathrm{~Hz}), 4.67(1 \mathrm{H}, \mathrm{d}, J=12.3 \mathrm{~Hz}), 4.75(1 \mathrm{H}, \mathrm{d}, J=12.6 \mathrm{~Hz}), 4.95(1 \mathrm{H}, \mathrm{d}, J=3 \mathrm{~Hz}), 7.26-$ $7.42(5 \mathrm{H}, \mathrm{m}) ;{ }^{13} \mathrm{C}$ NMR $\delta 18.0,18.0,48.1,48.2,63.4,65.4,66.1,68.2,69.5,97.1,100.3,100.5$, 127.8, 128.2, 128.5, 137.9; MS (ESI) $m / z$ (relative intensity) 377 ([M+Na $\left.]^{+}, 100\right)$; HRMS (ESI) calcd for $\mathrm{C}_{18} \mathrm{H}_{26} \mathrm{O}_{7}[\mathrm{M}+\mathrm{Na}]^{+}$377.1571, found 377.1572 . 

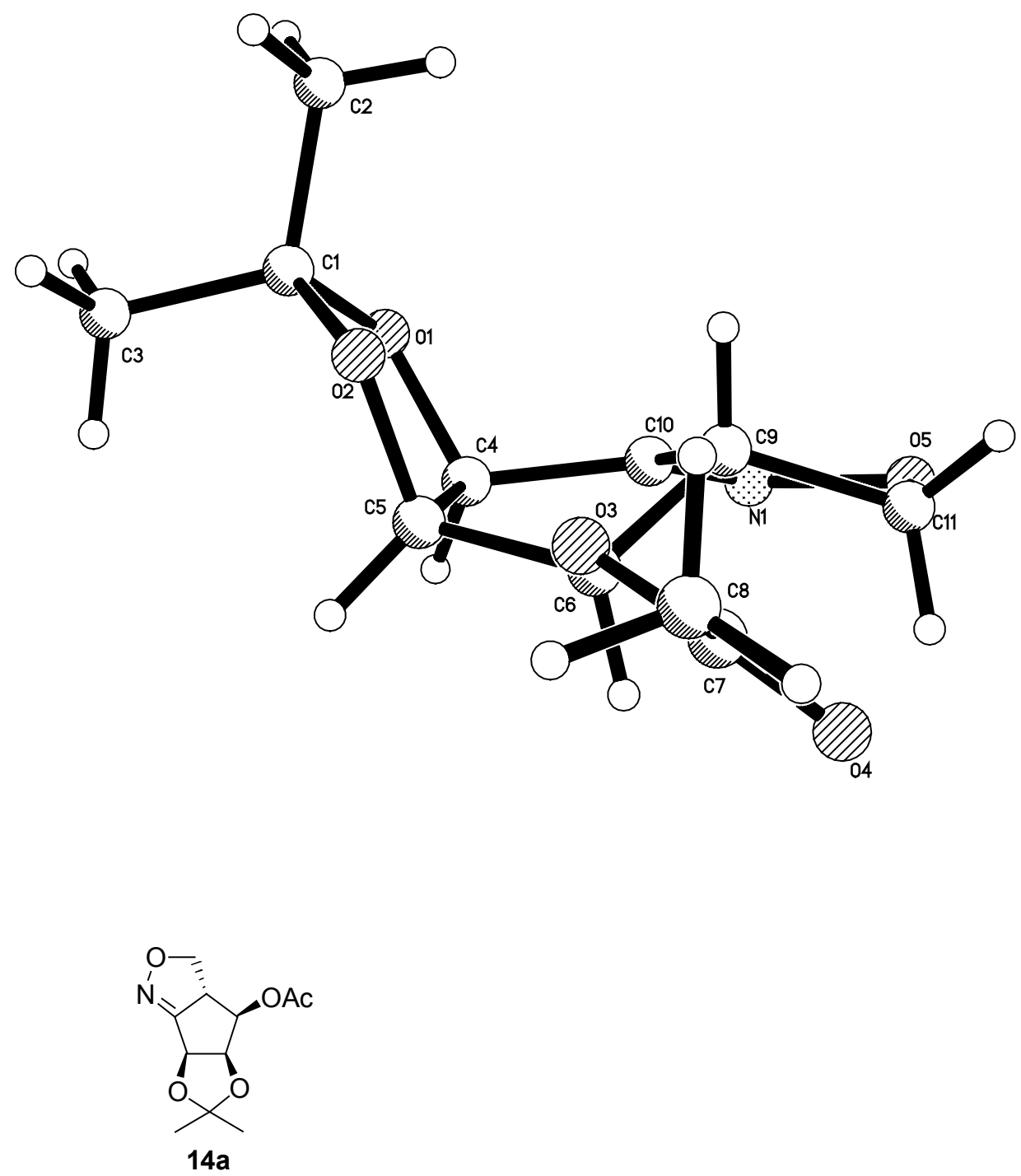

Figure 1. X-ray crystallographic structure of isoxazoline 14a. 

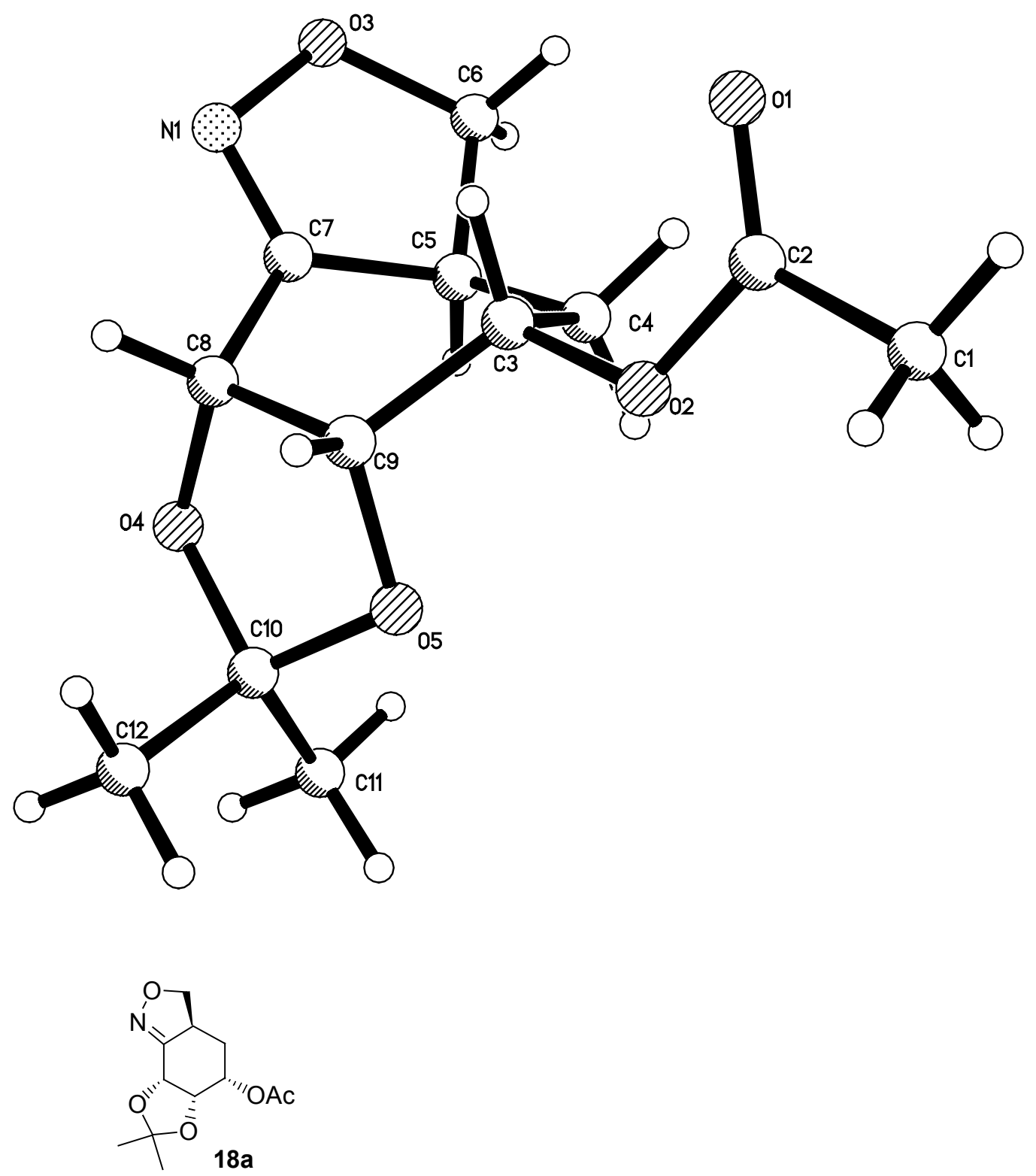

Figure 2. X-ray crystallographic structure of isoxazoline $18 \mathbf{a}$. 


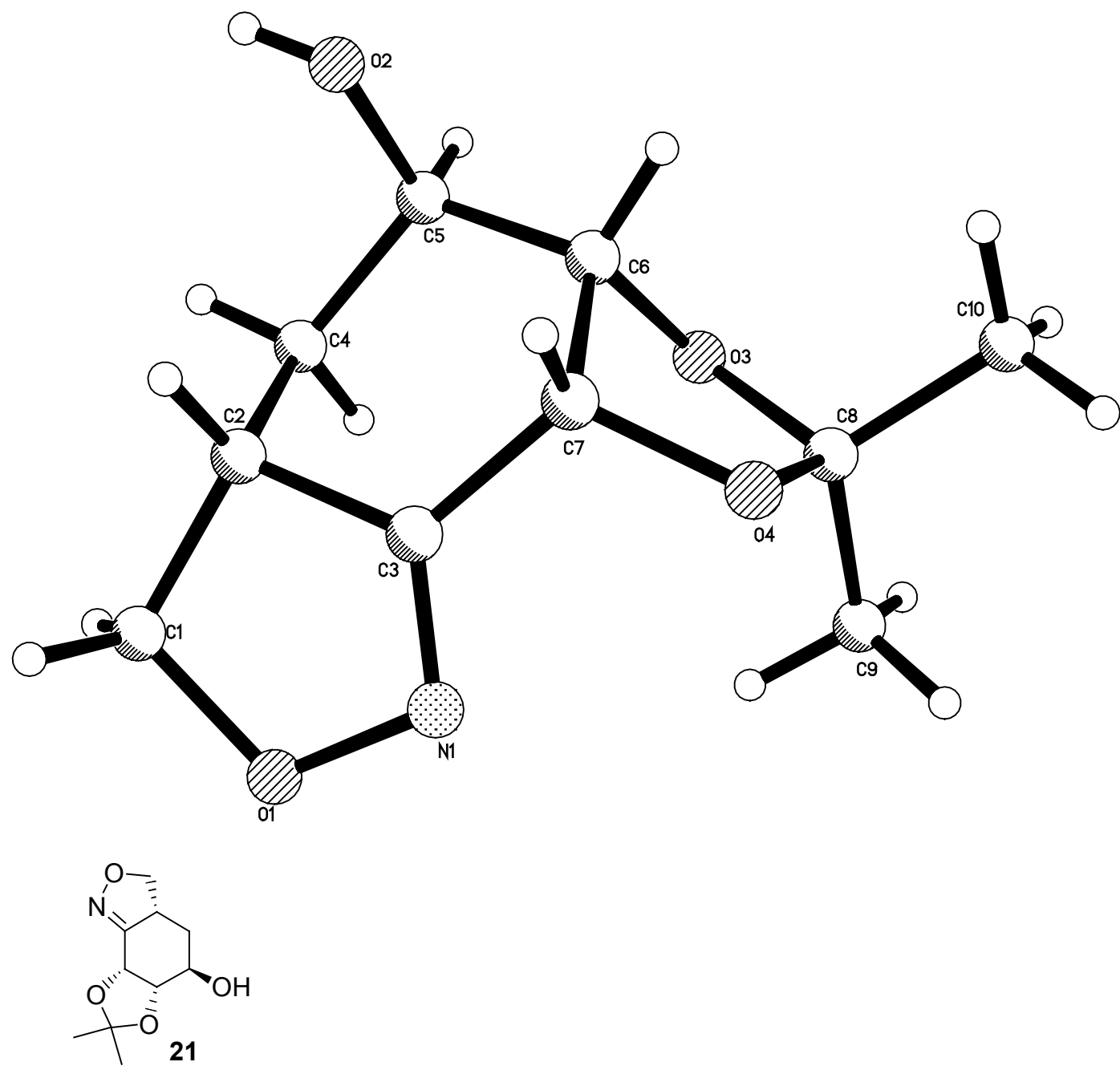

Figure 3. X-ray crystallographic structure of isoxazoline 21. 

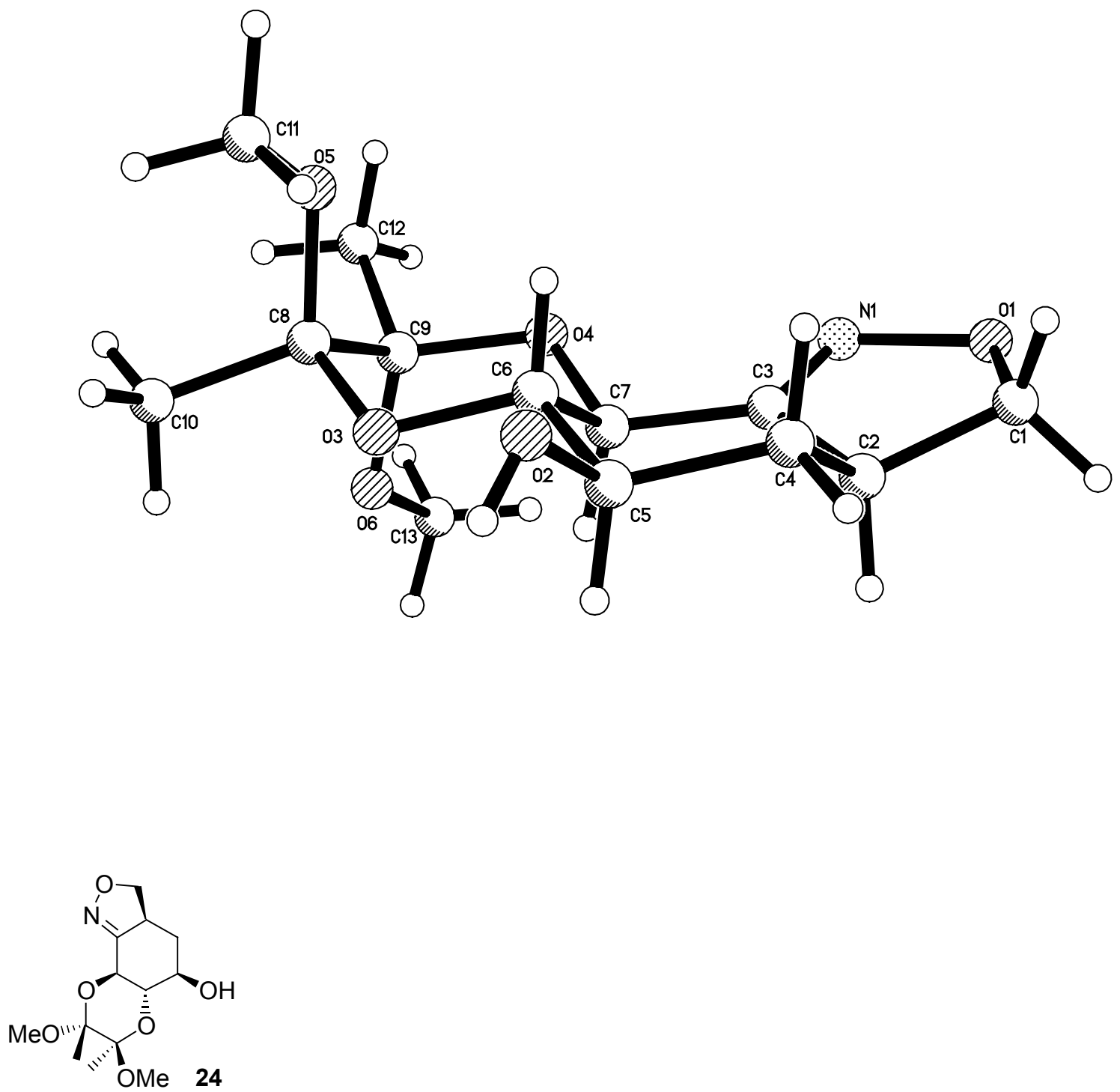

Figure 4. X-ray crystallographic structure of isoxazoline 24. 